\title{
Temperature Structures of Embedded Disks: Young Disks in Taurus Are Warm
}

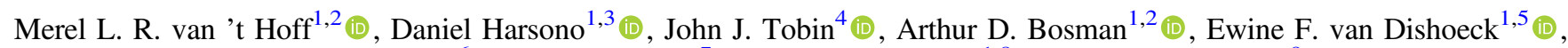 \\ Jes K. Jørgensen ${ }^{6}$ (D), Anna Miotello ${ }^{7}$ (D), Nadia M. Murillo ${ }^{1,8}$, and Catherine Walsh ${ }^{9}$ (D) \\ ${ }^{1}$ Leiden Observatory, Leiden University, P.O. Box 9513, 2300 RA Leiden, The Netherlands; mervth@umich.edu \\ ${ }^{2}$ Department of Astronomy, University of Michigan, 1085 South University Avenue, Ann Arbor, MI 48109, USA \\ ${ }^{3}$ Institute of Astronomy and Astrophysics, Academia Sinica, No. 1, Section 4, Roosevelt Road, Taipei 10617, Taiwan, R.O. C. \\ ${ }^{4}$ National Radio Astronomy Observatory, 520 Edgemont Road, Charlottesville, VA 22903, USA \\ ${ }^{5}$ Max-Planck-Institut für Extraterrestrische Physik, Giessenbachstrasse 1, D-85748 Garching bei München, Germany \\ ${ }^{6}$ Niels Bohr Institute, University of Copenhagen, Øster Voldgade 5-7, DK-1350 Copenhagen K., Denmark \\ ${ }^{7}$ European Southern Observatory, Karl-Schwarzschild-Str. 2, D-85748 Garching bei München, Germany \\ ${ }^{8}$ The Institute of Physical and Chemical Research (RIKEN), 2-1, Hirosawa, Wako-shi, Saitama 351-0198, Japan \\ ${ }^{9}$ School of Physics and Astronomy, University of Leeds, Leeds LS2 9JT, UK \\ Received 2020 July 13; revised 2020 August 17; accepted 2020 August 18; published 2020 October 6
}

\begin{abstract}
The chemical composition of gas and ice in disks around young stars sets the bulk composition of planets. In contrast to protoplanetary disks (Class II), young disks that are still embedded in their natal envelope (Class 0 and I) are predicted to be too warm for CO to freeze out, as has been confirmed observationally for L1527 IRS. To establish whether young disks are generally warmer than their more evolved counterparts, we observed five young (Class $0 / \mathrm{I}$ and I) disks in Taurus with the Atacama Large Millimeter/submillimeter Array, targeting $\mathrm{C}^{17} \mathrm{O} 2-1$, $\mathrm{H}_{2} \mathrm{CO} 3_{1,2}-2_{1,1}$, $\mathrm{HDO} 3_{1,2}-2_{2,1}$, and $\mathrm{CH}_{3} \mathrm{OH} 5_{K}-4_{K}$ transitions at 0 " $48 \times 0$ "' 31 resolution. The different freeze-out temperatures of these species allow us to derive a global temperature structure. $\mathrm{C}^{17} \mathrm{O}$ and $\mathrm{H}_{2} \mathrm{CO}$ are detected in all disks, with no signs of $\mathrm{CO}$ freeze-out in the inner $\sim 100$ au and a $\mathrm{CO}$ abundance close to $\sim 10^{-4}$. The $\mathrm{H}_{2} \mathrm{CO}$ emission originates in the surface layers of the two edge-on disks, as witnessed by the especially beautiful V-shaped emission pattern in IRAS $04302+2247$. $\mathrm{HDO}$ and $\mathrm{CH}_{3} \mathrm{OH}$ are not detected, with column density upper limits more than 100 times lower than for hot cores. Young disks are thus found to be warmer than more evolved protoplanetary disks around solar analogs, with no CO freeze-out (or only in the outermost part of $\gtrsim 100$ au disks) or processing. However, they are not as warm as hot cores or disks around outbursting sources and therefore do not have a large gas-phase reservoir of complex molecules.
\end{abstract}

Unified Astronomy Thesaurus concepts: Protostars (1302); Protoplanetary disks (1300); Young stellar objects (1834); Astrochemistry (75)

\section{Introduction}

Disks around young stars provide the material from which planets form. Knowledge of their physical and chemical structure is therefore crucial for understanding planet formation and composition. The physics of protoplanetary disks has been studied in great detail, both using observations of individual objects (e.g., van Zadelhoff et al. 2001; Andrews et al. 2010; Andrews et al. 2018; Schwarz et al. 2016) and through surveys of star-forming regions (e.g., Ansdell et al. 2016, 2017; Barenfeld et al. 2016; Pascucci et al. 2016; Cox et al. 2017; Ruíz-Rodríguez et al. 2018; Cieza et al. 2019). Molecular line observations require more telescope time than continuum observations; hence, studies of the chemical structure generally target individual disks or small samples of bright disks (e.g., Dutrey et al. 1997; Thi et al. 2004; Öberg et al. 2010; Cleeves et al. 2015; Huang et al. 2017). The picture that is emerging for the global composition of Class II disks around solar analogs is that they have a large cold outer region $(T \lesssim 20 \mathrm{~K})$ where $\mathrm{CO}$ is frozen out in the disk midplanes (e.g., Aikawa et al. 2002; Mathews et al. 2013; Qi et al. 2013b, 2015, 2019; Dutrey et al. 2017).

However, it is now becoming clear that planet formation already starts when the disk is still embedded in its natal envelope. Grain growth has been observed in Class 0 and I sources, and even larger bodies may have formed before the envelope has fully dissipated (e.g., Jørgensen et al. 2009; Kwon et al. 2009; Miotello et al. 2014; ALMA Partnership et al. 2015;
Harsono et al. 2018). Furthermore, the dust mass of Class II disks seems insufficient to form the observed exoplanet population, but Class 0 and I disks are massive enough (Manara et al. 2018; Tychoniec et al. 2020). Young embedded disks thus provide the initial conditions for planet formation, but unlike their more evolved counterparts, their structure remains poorly characterized.

A critical property is the disk temperature structure, because this governs disk evolution and composition. For example, temperature determines whether the gas is susceptible to gravitational instabilities (see, e.g., a review by Kratter \& Lodato 2016), a potential mechanism to form giant planets, stellar companions, and accretion bursts (e.g., Boss 1997; Boley 2009; Vorobyov 2009; Tobin et al. 2016a). In addition, grain growth is thought to be enhanced in the region where water freezes out from the gas phase onto the dust grains, the water snowline $(T \sim 100-150 \mathrm{~K}$; e.g., Stevenson \& Lunine 1988; Drążkowska \& Alibert 2017; Schoonenberg \& Ormel 2017).

Moreover, freeze-out of molecules as the temperature drops below their species-specific freeze-out temperature sets the global chemical composition of the disk. This sequential freeze-out causes radial gradients in molecular abundances and elemental ratios (like the $\mathrm{C} / \mathrm{O}$ ratio; e.g., Öberg et al. 2011). In turn, the composition of a planet then depends on its formation location in the disk (e.g., Madhusudhan et al. 2014; Walsh et al. 2015; Ali-Dib 2017; Cridland et al. 2019). Finally, 
the formation of high abundances of complex molecules starts from CO ice (e.g., Tielens \& Hagen 1982; Garrod \& Herbst 2006; Cuppen et al. 2009; Chuang et al. 2016), and COM formation will thus be impeded during the disk stage if the temperature is above the $\mathrm{CO}$ freeze-out temperature $(T \gtrsim 20 \mathrm{~K})$. Whether young disks are warm $(T \gtrsim 20 \mathrm{~K}$; i.e., warmer than the $\mathrm{CO}$ freeze-out temperature) or cold (i.e., have a large region where $T \lesssim 20 \mathrm{~K}$ and $\mathrm{CO}$ is frozen out) is thus a simple but crucial question.

Keplerian disks are now detected around several Class 0 and I sources (e.g., Brinch et al. 2007; Tobin et al. 2012; Murillo et al. 2013; Yen et al. 2017), but most research has focused on disk formation, size, and kinematics (e.g., Yen et al. 2013; Harsono et al. 2014; Ohashi et al. 2014) or the chemical structure at the disk-envelope interface (e.g., Sakai et al. 2014b; Murillo et al. 2015; Oya et al. 2016). Only a few studies have examined the disk physical structure, and only for one particular disk, L1527 IRS. Tobin et al. (2013) and Aso et al. (2017) modeled the radial density profile, and van 't Hoff et al. (2018a) studied its temperature profile based on optically thick ${ }^{13} \mathrm{CO}$ and $\mathrm{C}^{18} \mathrm{O}$ observations. The latter study showed the importance of disentangling disk and envelope emission and concluded that the entire L1527 disk is likely too warm for CO freeze-out, in agreement with model predictions (e.g., Harsono et al. 2015) but in contrast to observations of $\mathrm{T}$ Tauri disks.

Another important question with regard to the composition of planet-forming material is the $\mathrm{CO}$ abundance. The majority of protoplanetary disks have surprisingly weak $\mathrm{CO}$ emission, even when freeze-out and isotope-selective photodissociation are taken into account (e.g., Ansdell et al. 2016; Long et al. 2017; Miotello et al. 2017). Based on gas masses derived from HD line fluxes (Favre et al. 2013; Kama et al. 2016; McClure et al. 2016; Schwarz et al. 2016) and mass accretion rates (Manara et al. 2016) the low CO emission seems to be the result of significant $\mathrm{CO}$ depletion (up to 2 orders of magnitude below the interstellar medium (ISM) abundance of $\sim 10^{-4}$ with respect to $\mathrm{H}_{2}$ ).

Several mechanisms have been discussed in the literature, either focusing on the chemical conversion of $\mathrm{CO}$ into less volatile species (e.g., Bergin et al. 2014; Eistrup et al. 2016; Bosman et al. 2018; Schwarz et al. 2018, 2019) or using dust growth to sequester $\mathrm{CO}$ ice in the disk midplane (e.g., $\mathrm{Xu}$ et al. 2017; Krijt et al. 2018). Observations of CO abundances in younger disks can constrain the timescale of the $\mathrm{CO}$ depletion process. Observations of ${ }^{13} \mathrm{CO}$ and $\mathrm{C}^{18} \mathrm{O}$ toward the embedded sources TMC1A and L1527 are consistent with an ISM abundance (Harsono et al. 2018; van 't Hoff et al. 2018a). Recent work by Zhang et al. (2020) also found CO abundances consistent with the ISM abundance for three young disks in Taurus with ages up to $\sim 1$ Myr using optically thin ${ }^{13} \mathrm{C}^{18} \mathrm{O}$ emission. Since the 2-3 Myr old disks in Lupus and Cha I show CO depletion by a factor of 10-100 (Ansdell et al. 2016), these results suggest that the $\mathrm{CO}$ abundance decreases by a factor of 10 within 1 Myr. On the other hand, Bergner et al. (2020) found $\mathrm{C}^{18} \mathrm{O}$ abundances a factor of 10 below the ISM value in two Class I sources in Serpens.

In this paper, we present Atacama Large Millimeter/ submillimeter Array (ALMA) observations of $\mathrm{C}^{17} \mathrm{O}$ toward five young disks in Taurus to address the questions of whether young disks are generally too warm for $\mathrm{CO}$ freeze-out and whether there is significant $\mathrm{CO}$ processing. The temperature profile is further constrained by $\mathrm{H}_{2} \mathrm{CO}$ observations, as this molecule freezes out around $\sim 70 \mathrm{~K}$. Although chemical models often assume a binding energy of $2050 \mathrm{~K}$ (e.g., Garrod \& Herbst 2006; McElroy et al. 2013), laboratory experiments have found binding energies ranging between 3300 and $3700 \mathrm{~K}$, depending on the ice surface (Noble et al. 2012). These latter values suggest $\mathrm{H}_{2} \mathrm{CO}$ freeze-out temperatures between $\sim 70$ and $90 \mathrm{~K}$ for disk midplane densities $\left(\sim 10^{8}-10^{10} \mathrm{~cm}^{-3}\right)$ instead of $\sim 50 \mathrm{~K}$. Experiments by Fedoseev et al. (2015) are consistent with the lower end of binding energies found by Noble et al. (2012), so we adopt a freeze-out temperature of $70 \mathrm{~K}$ for $\mathrm{H}_{2} \mathrm{CO}$. An initial analysis of these observations was presented in van 't Hoff (2019).

In addition, $\mathrm{HDO}$ and $\mathrm{CH}_{3} \mathrm{OH}$ observations are used to probe the $\gtrsim 100-150 \mathrm{~K}$ region and determine whether complex molecules can be observed in these young disks, as shown for the disk around the outbursting young star V883 Ori (van 't Hoff et al. 2018b; Lee et al. 2019). In contrast, observing complex molecules has turned out to be very difficult in mature protoplanetary disks. So far, only $\mathrm{CH}_{3} \mathrm{CN}$ has been detected in a sample of disks, and $\mathrm{CH}_{3} \mathrm{OH}$ and $\mathrm{HCOOH}$ have been detected in TW Hya (Öberg et al. 2015; Walsh et al. 2016; Bergner et al. 2018; Favre et al. 2018; Loomis et al. 2018; Carney et al. 2019).

The observations are described in Section 2, and the resulting $\mathrm{C}^{17} \mathrm{O}$ and $\mathrm{H}_{2} \mathrm{CO}$ images are presented in Section 3. This section also describes the nondetections of HDO and $\mathrm{CH}_{3} \mathrm{OH}$. The temperature structure of the disks is examined in Section 4 based on the $\mathrm{C}^{17} \mathrm{O}$ and $\mathrm{H}_{2} \mathrm{CO}$ observations and radiative transfer modeling. The result that the young disks in this sample are warm with no significant $\mathrm{CO}$ freeze-out or processing is discussed in Section 5 and the conclusions are summarized in Section 6.

\section{Observations}

In order to study the temperature structure in young disks, a sample of five Class I protostars in Taurus was observed with ALMA: IRAS 04302+2247 (also known as the Butterfly star, hereafter IRAS 04302), L1489 IRS (hereafter L1489), L1527 IRS (hereafter L1527), TMC1, and TMC1A. All sources are known to have a disk, and Keplerian rotation has been established (Brinch et al. 2007; Tobin et al. 2012; Harsono et al. 2014, M. L. R. van 't Hoff et al. 2020, in preparation). The objects IRAS 04302 and L1527 are seen edge-on, which allows a direct view of the midplane, whereas L1489, TMC1, and TMC1A are moderately inclined by $\sim 50^{\circ}-60^{\circ}$. The source properties are listed in Table 1.

The observations were carried out on 2018 September 10 and 28 with a total on-source time of 15 minutes per source (project code 2017.1.01413.S). The observations used 47 antennas sampling baselines between $15 \mathrm{~m}$ and $1.4 \mathrm{~km}$. The correlator setup included a $2 \mathrm{GHz}$ continuum band with $488 \mathrm{kHz}\left(0.6 \mathrm{~km} \mathrm{~s}^{-1}\right)$ resolution centered at $240.0 \mathrm{GHz}$ and spectral windows targeting $\mathrm{C}^{17} \mathrm{O} 2-1, \mathrm{H}_{2} \mathrm{CO} 33_{1,2}-2_{1,1}$, HDO $3_{1,2}-2_{2,1}$, and several $\mathrm{CH}_{3} \mathrm{OH} 5_{K}-4_{K}$ transitions. The spectral resolution was $122.1 \mathrm{kHz}$ for $\mathrm{CH}_{3} \mathrm{OH}$ and $61.0 \mathrm{kHz}$ for the other lines, which corresponds to a velocity resolution of 0.15 and $0.08 \mathrm{~km} \mathrm{~s}^{-1}$, respectively. The properties of the targeted lines can be found in Table A1.

Calibration was done using the ALMA pipeline and version 5.4.0 of the Common Astronomy Software Applications (CASA; McMullin et al. 2007). The phase calibrator was J0438+3004, and the bandpass and flux calibrator was J0510+1800. In addition, we performed up to three rounds of phase-only self- 
Table 1

Overview of Source Properties

\begin{tabular}{|c|c|c|c|c|c|c|c|c|c|c|c|c|}
\hline $\begin{array}{l}\text { Source Name } \\
\text { (IRAS) }\end{array}$ & Other Name & $\begin{array}{l}\text { R.A. }^{\mathrm{a}} \\
(\mathrm{J} 2000)\end{array}$ & $\begin{array}{l}\text { Decl. }^{\mathrm{a}} \\
(\mathrm{J} 2000)\end{array}$ & Class & $\begin{array}{l}T_{\text {bol }} \\
(\mathrm{K})\end{array}$ & $\begin{array}{l}L_{\mathrm{bol}} \\
\left(L_{\odot}\right)\end{array}$ & $\begin{array}{c}M_{*} \\
\left(M_{\odot}\right)\end{array}$ & $\begin{array}{l}M_{\text {env }} \\
\left(M_{\odot}\right)\end{array}$ & $\begin{array}{l}M_{\text {disk }} \\
\left(M_{\odot}\right)\end{array}$ & $\begin{array}{c}R_{\text {disk }} \\
(\mathrm{au})\end{array}$ & $\begin{array}{c}i \\
\text { (deg) }\end{array}$ & Refs. $^{\text {b }}$ \\
\hline $04016+2610$ & L1489 IRS & 04:04:43.1 & $+26: 18: 56.2$ & I & 226 & 3.5 & 1.6 & 0.023 & 0.0071 & 600 & 66 & $1-4$ \\
\hline $04302+2247$ & Butterfly star & $04: 33: 16.5$ & $+22: 53: 20.4$ & $\mathrm{I} / \mathrm{II}$ & 202 & $0.34-0.92$ & $0.5^{\mathrm{c}}$ & 0.017 & 0.11 & 244 & $>76$ & $3,5,9$ \\
\hline $04368+2557$ & L1527 IRS & 04:39:53.9 & $+26: 03: 09.5$ & $0 / \mathrm{I}$ & 59 & $1.9-2.75$ & $0.19-0.45$ & $0.9-1.7$ & 0.0075 & $75-125$ & 85 & $9-14$ \\
\hline $04381+2540$ & TMC1 & $04: 41: 12.7$ & $+25: 46: 34.8$ & I & 171 & $0.66-0.9$ & 0.54 & 0.14 & 0.0039 & 100 & 55 & $1,6,10$ \\
\hline
\end{tabular}

Notes. All values presented in this table are from the literature listed in footnote b. Here TMC1 is resolved for the first time as a binary. The literature values in this table are derived assuming a single source.

${ }^{a}$ Peak of the continuum emission, except for TMC1, where the phase center of the observations is listed. The coordinates of the two sources TMC1-E and TMC1-W are R.A. $=04: 41: 12.73$, decl. $=+25: 46: 34.76$ and R.A. $=04: 41: 12.69$, decl. $=+25: 46: 34.73$, respectively.

${ }^{\mathrm{b}}$ References. (1) Green et al. (2013), (2) Yen et al. (2014), (3) Sheehan \& Eisner (2017), (4) Sai et al. (2020), (5) Wolf et al. (2003), (6) Harsono et al. (2014), (7) Aso et al. (2015), (8) Harsono et al., submitted, (9) Motte \& André (2001), (10) Kristensen et al. (2012), (11) Tobin et al. (2008), (12) Tobin et al. (2013), (13) Oya et al. (2015), (14) Aso et al. (2017).

${ }^{\mathrm{c}}$ Not a dynamical mass.

Table 2

Observed Fluxes for the $1.3 \mathrm{~mm}$ Continuum and Molecular Lines

\begin{tabular}{|c|c|c|c|c|}
\hline Source & $\begin{array}{c}F_{\text {peak }}(1.3 \mathrm{~mm}) \\
\left(\mathrm{mJy}^{-1} \text { beam }^{-1}\right)\end{array}$ & $\begin{array}{c}F_{\text {int }} \underset{(\mathrm{mJy})}{(1.3 \mathrm{~mm})} \\
\end{array}$ & $\begin{array}{l}F_{\text {int }}\left(\mathrm{C}^{17} \mathrm{O}\right)^{\mathrm{a}} \\
\left(\mathrm{Jy} \mathrm{km} \mathrm{s}^{-1}\right)\end{array}$ & $\begin{array}{r}F_{\text {int }}\left(\mathrm{H}_{2} \mathrm{CO}\right)^{\mathrm{a}} \\
\left(\mathrm{Jy} \mathrm{km} \mathrm{s}^{-1}\right)\end{array}$ \\
\hline IRAS $04302+2247$ & $24.7 \pm 0.1$ & $165.9 \pm 0.8$ & $2.2 \pm 0.2$ & $3.5 \pm 0.2$ \\
\hline L1489 IRS & $2.8 \pm 0.1$ & $51.1 \pm 1.1$ & $2.9 \pm 0.3$ & $8.0 \pm 0.5$ \\
\hline L1527 IRS & $102.0 \pm 0.1$ & $195.1 \pm 0.4$ & $1.9 \pm 0.4$ & $3.0 \pm 0.6$ \\
\hline TMC1A & $125.8 \pm 0.2$ & $210.4 \pm 0.4$ & $4.1 \pm 0.4$ & $2.3 \pm 0.2$ \\
\hline TMC1-E & $9.2 \pm 0.1$ & $10.3 \pm 0.2$ & $2.0 \pm 0.3^{\mathrm{b}}$ & $2.6 \pm 0.2^{\mathrm{b}}$ \\
\hline TMC1-W & $16.2 \pm 0.1$ & $17.6 \pm 0.2$ & $2.0 \pm 0.3^{\mathrm{b}}$ & $2.6 \pm 0.2^{\mathrm{b}}$ \\
\hline
\end{tabular}

Notes. The listed errors are statistical errors and do not include calibration uncertainties.

a Integrated flux within a circular aperture with $66^{\prime \prime} 0$ diameter.

${ }^{\mathrm{b}}$ Flux for both sources together.

calibration on the continuum data with solution intervals that spanned the entire scan length for the first round, as short as $60 \mathrm{~s}$ in the second round, and as short as $30 \mathrm{~s}$ in the third round. The obtained phase solutions were also applied to the line data. Imaging was done using tclean in CASA version 5.6.1. The typical restoring beam size using Briggs weighting with a robust parameter of 0.5 is 0 ." $42 \times 0$ ". $28(59 \times 39 \mathrm{au})$ for the continuum images and 0 ". $48 \times 0$ ". $31(67 \times 43 \mathrm{au})$ for the line images. The continuum images have an $\mathrm{rms}$ of $\sim 0.07 \mathrm{mJy}^{-1}$ beam $^{-1}$, whereas the rms in the line images is $\sim 5 \mathrm{mJybeam}^{-1}$ channel $^{-1}$ for $0.08 \mathrm{~km} \mathrm{~s}^{-1}$ channels. The observed continuum and line flux densities are reported in Table 2.

\section{Results \\ 3.1. $\mathrm{C}^{17} \mathrm{O}$ and $\mathrm{H}_{2} \mathrm{CO}$ Morphology}

Figure 1 shows the $1.3 \mathrm{~mm}$ continuum images and integrated intensity (zeroth moment) maps for $\mathrm{C}^{17} \mathrm{O} 2-1$ and $\mathrm{H}_{2} \mathrm{CO}$ $3_{1,2}-2_{1,1}$ toward the five sources in our sample. The molecular emission toward IRAS 04302 is highlighted at slightly higher spatial resolution in Figure 2. Radial cuts along the major axis are presented in Figure 3. The continuum emission is elongated perpendicular to the outflow direction for all sources, consistent with a disk as observed before. For the first time, TMC1 is resolved into a close binary ( $\sim 85$ au separation). We will refer to the two sources as TMC1-E (east) and TMC1-W (west).

Both $\mathrm{C}^{17} \mathrm{O}$ and $\mathrm{H}_{2} \mathrm{CO}$ are clearly detected toward all sources with a velocity gradient along the continuum structures (see
Figure A1). The velocity gradient suggests that the material in TMC1 is located in a circumbinary disk, but a detailed analysis is beyond the scope of this paper. For both molecules, integrated fluxes are similar (within a factor of 2-3) in all sources (Table 2), and both lines have a comparable (factor of 2-4) strength toward each source, with $\mathrm{H}_{2} \mathrm{CO}$ brighter than $\mathrm{C}^{17} \mathrm{O}$, except for TMC1A. The $\mathrm{H}_{2} \mathrm{CO}$ emission is generally more extended than the $\mathrm{C}^{17} \mathrm{O}$ emission, both radially and vertically, except toward TMC1 and TMC1A, where both molecules have the same spatial extent. This is not a signal-tonoise issue, as can be seen from the radial cuts along the major axis (Figure 3).

The most striking feature in the integrated intensity maps is the $\mathrm{V}$-shaped emission pattern of the $\mathrm{H}_{2} \mathrm{CO}$ in the edge-on disk IRAS 04302 (see Figure 2), suggesting that the emission arises from the disk surface layers and not the midplane, in contrast to the $\mathrm{C}^{17} \mathrm{O}$ emission. The $\mathrm{H}_{2} \mathrm{CO}$ emission displays a ringlike structure toward L1527. Given that this disk is also viewed edgeon, this can be explained by emission originating in the disk surface layers, with the outer component along the midplane arising from the envelope. As we will show later in this section, the emission toward IRAS 04302 shows very little envelope contribution, which can explain the difference in morphology between these two sources. The $\mathrm{C}^{17} \mathrm{O}$ emission peaks slightly offset ( $\sim 60 \mathrm{au}$ ) from the L1527 continuum peak, probably due to the dust becoming optically thick in the inner $\sim 10 \mathrm{au}$, as seen before for ${ }^{13} \mathrm{CO}$ and $\mathrm{C}^{18} \mathrm{O}$ (van 't Hoff et al. 2018a). The current resolution does not resolve the inner $10 \mathrm{au}$; hence, the reduction in CO emission is more extended. In IRAS 04302, a similar offset of 
IRAS 04302
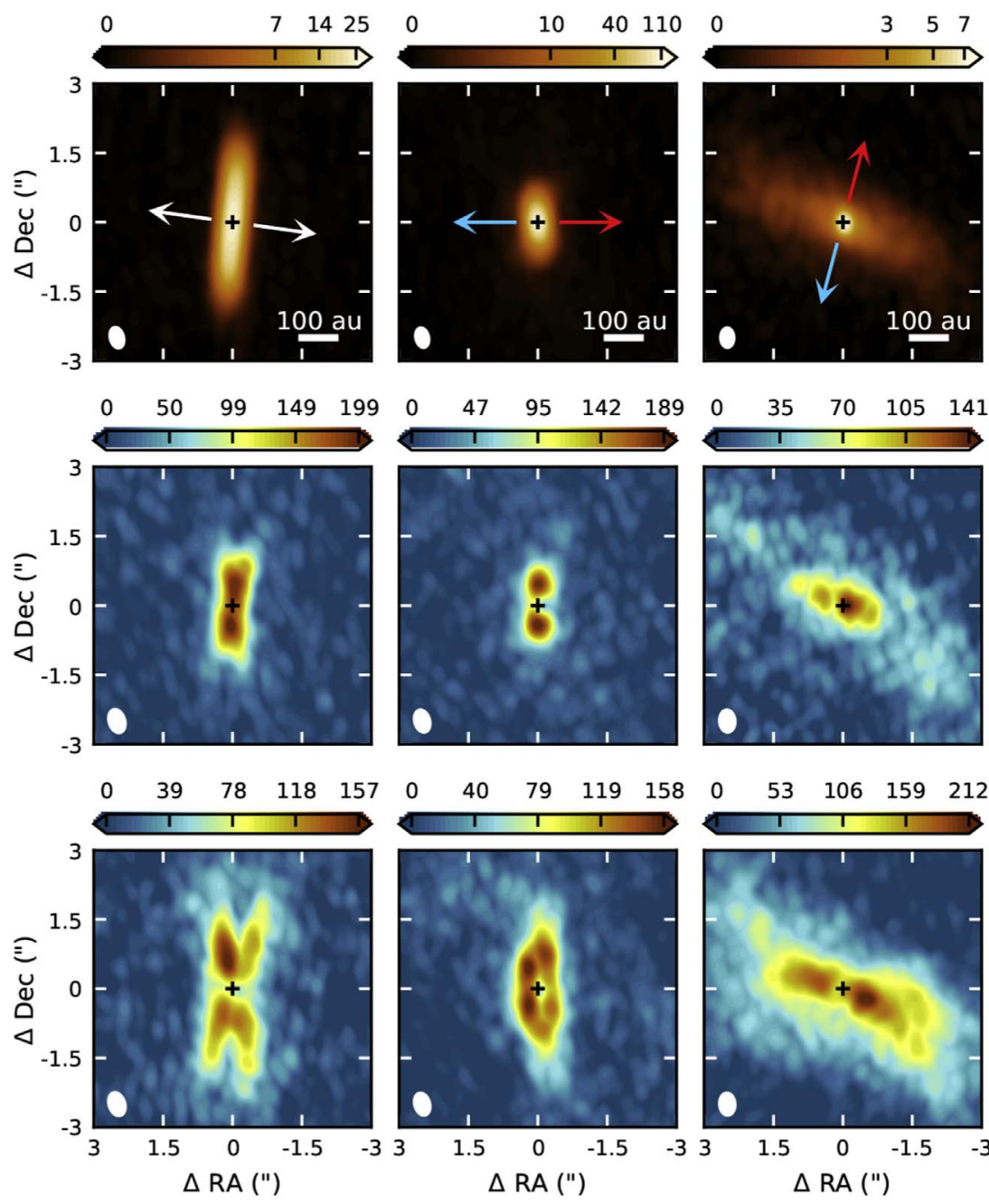

L1489

TMC1A

TMC1
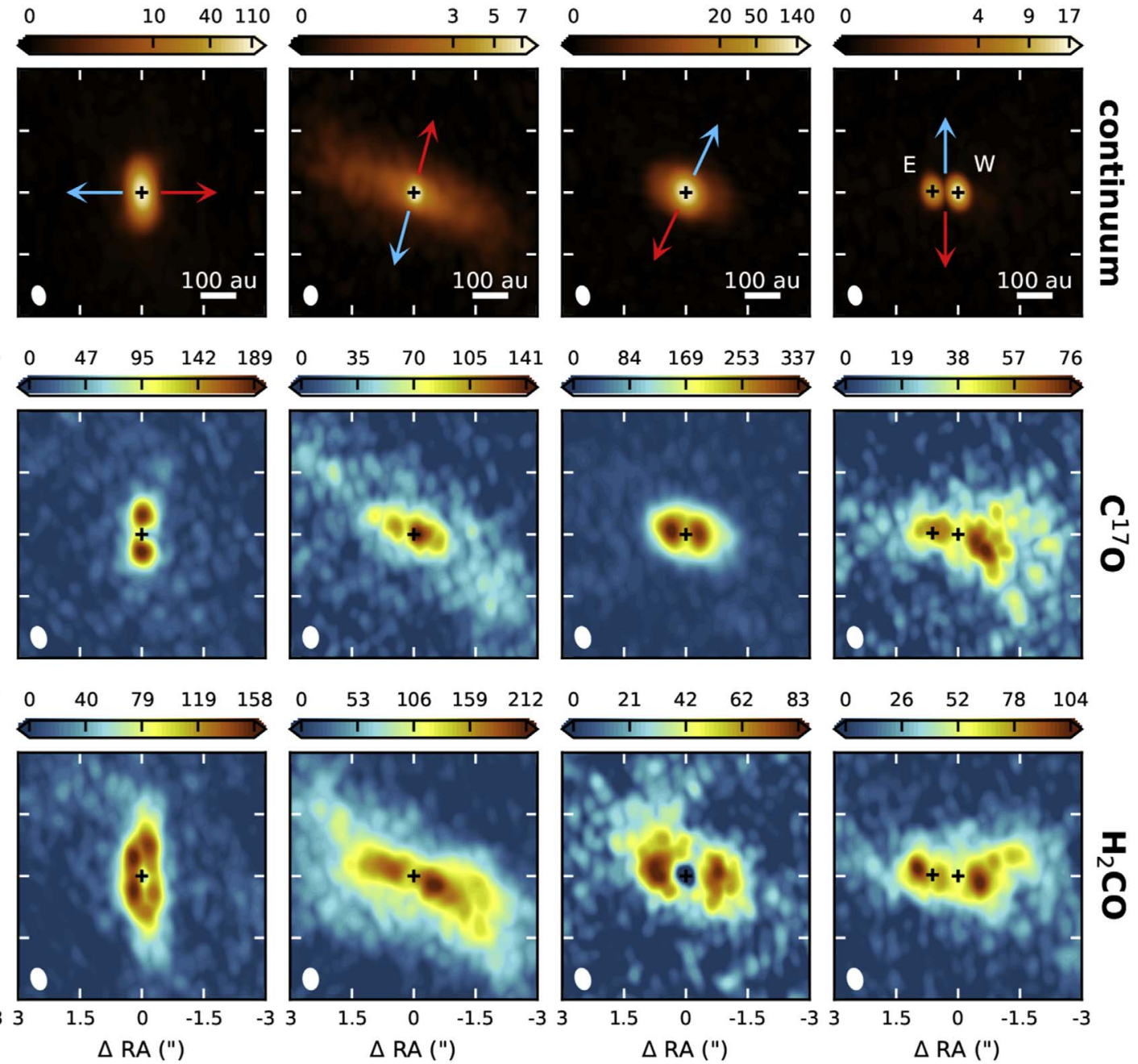

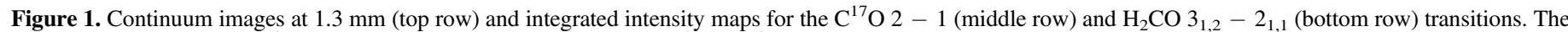

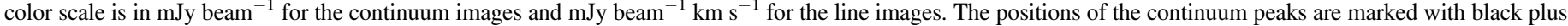
signs, and the outflow directions are indicated by arrows in the continuum images. The beam is shown in the lower left corner of each panel.

$\sim 60$ au is found for both $\mathrm{C}^{17} \mathrm{O}$ and $\mathrm{H}_{2} \mathrm{CO}$, suggesting that there may be an unresolved optically thick dust component as well.

Toward L1489, $\mathrm{C}^{17} \mathrm{O}$ has a bright inner component $(\sim 200$ $\mathrm{au}$ ) and a weaker outer component that extends roughly as far as the $\mathrm{H}_{2} \mathrm{CO}$ emission $(\sim 600 \mathrm{au})$. A similar structure was observed in $\mathrm{C}^{18} \mathrm{O}$ by Sai et al. (2020). The slight rise seen in $\mathrm{C}^{18} \mathrm{O}$ emission around $\sim 300$ au to the southwest of the continuum peak is also visible in the $\mathrm{C}^{17} \mathrm{O}$ radial cut. Imaging the $\mathrm{C}^{17} \mathrm{O}$ data at lower resolution makes this feature clearer in the integrated intensity map. In contrast, the $\mathrm{H}_{2} \mathrm{CO}$ emission decreases in the inner $\sim 75 \mathrm{au}$, but beyond that, it extends smoothly out to $\sim 600 \mathrm{au}$. The off-axis protrusions at the outer edge of the disk pointing to the northeast and southwest were also observed in $\mathrm{C}^{18} \mathrm{O}$ and explained as streams of infalling material (Yen et al. 2014).

The $\mathrm{C}^{17} \mathrm{O}$ emission peaks slightly ( $\left.\sim 40-50 \mathrm{au}\right)$ off source toward TMC1A. Harsono et al. (2018) showed that ${ }^{13} \mathrm{CO}$ and $\mathrm{C}^{18} \mathrm{O}$ emission is absent in the inner $\sim 15$ au due to the dust being optically thick. The resolution of the $\mathrm{C}^{17} \mathrm{O}$ observations is not high enough to resolve this region, resulting in only a central decrease in emission instead of a gap. A clear gap is visible for $\mathrm{H}_{2} \mathrm{CO}$ with the emission peaking $\sim 100-115$ au off source. The central absorption falling below zero is an effect of resolved-out large-scale emission.

Finally, toward TMC1, $\mathrm{H}_{2} \mathrm{CO}$ shows a dip at both continuum peaks, while the $\mathrm{C}^{17} \mathrm{O}$ emission is not affected by the eastern continuum peak. As discussed for the other sources, this may be the result of optically thick dust in the inner disk. The protrusions seen on the west side in both $\mathrm{C}^{17} \mathrm{O}$ and $\mathrm{H}_{2} \mathrm{CO}$ are part of a larger arc-like structure that extends toward the southwest beyond the scale shown in the image.

While it is tempting to ascribe all of the compact emission to the young disk, some of it may also come from the envelope and obscure the disk emission. To get a first impression as to whether the observed emission originates in the disk or envelope, position-velocity ( $p v)$ diagrams are constructed along the disk major axis for the four single sources (Figure 4). In these diagrams, disk emission is located at small angular offsets and high velocities, while envelope emission extends to larger offsets but has lower velocities. In all sources, $\mathrm{C}^{17} \mathrm{O}$ predominantly traces the disk, with some envelope contribution, especially in L1527 and L1489. The $\mathrm{H}_{2} \mathrm{CO}$ emission also originates in the disk but has a larger envelope component. An exception is IRAS 04302, which shows hardly 


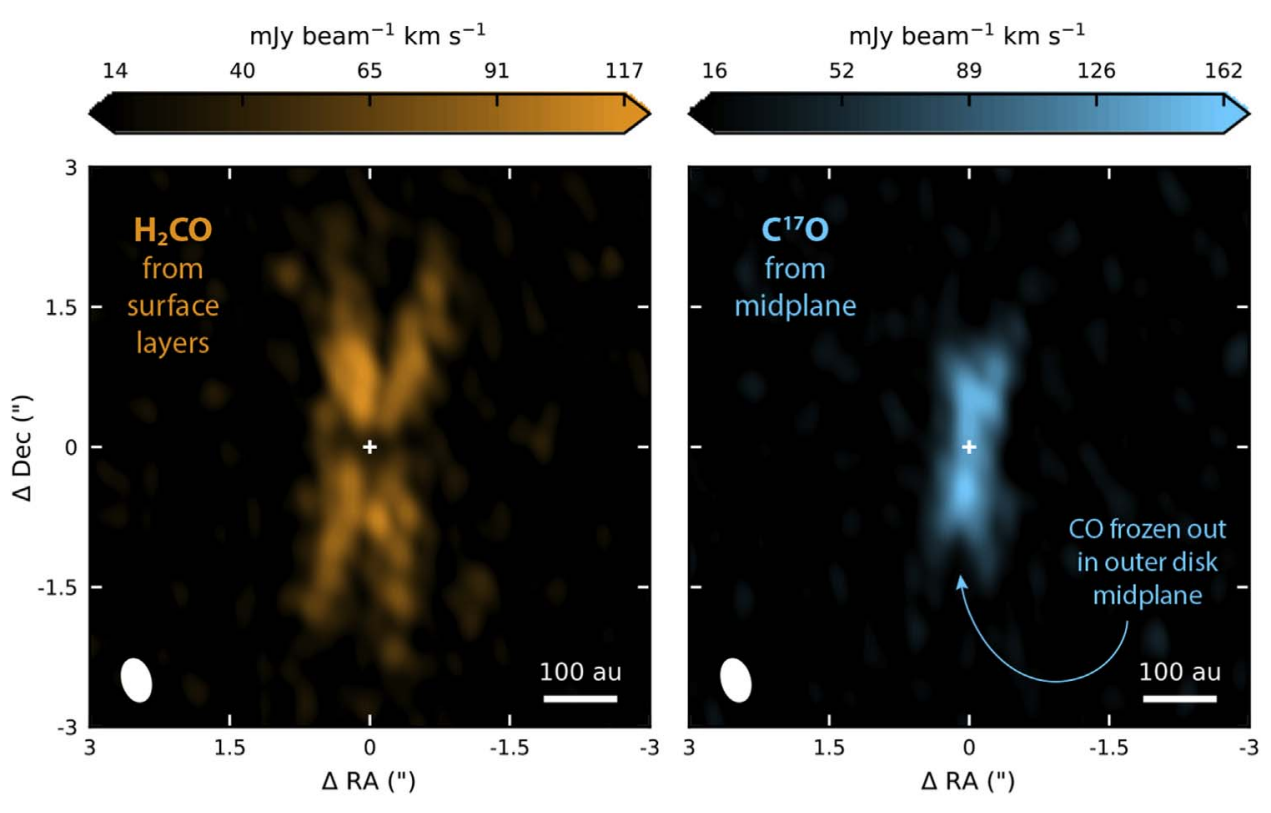

Figure 2. Integrated intensity maps for the $\mathrm{H}_{2} \mathrm{CO}_{1,2}-2_{1,1}$ (left) and $\mathrm{C}^{17} \mathrm{O} 2-1$ (right) emission toward IRAS 04302 . These images have slightly higher resolution than shown in Figure $1(0 !$ " $45 \times 0$.' 28$)$ due to uniform weighting of the visibilities. The positions of the continuum peaks are marked with white plus signs, and the beam is shown in the lower left corner of each panel.

any envelope contribution. These results for L1527 are in agreement with previous observations (Sakai et al. 2014a). In L1489, a bright linear feature is present for $\mathrm{H}_{2} \mathrm{CO}$ extending from a velocity and angular offset of $-2 \mathrm{~km} \mathrm{~s}^{-1}$ and $-2^{\prime \prime}$, respectively, to offsets of $2 \mathrm{~km} \mathrm{~s}^{-1}$ and $2^{\prime \prime}$. This feature matches the shape of the SO $p v$ diagram (Yen et al. 2014), which was interpreted by the authors as a ring between $\sim 250$ and 390 au. While a brightness enhancement was also identified by Yen et al. (2014) in the $\mathrm{C}^{18} \mathrm{O}$ emission (similar to that seen here for $\mathrm{H}_{2} \mathrm{CO}$ ), the $\mathrm{C}^{17} \mathrm{O}$ emission does not display such a feature.

Another way to determine the envelope contribution is from the visibility amplitudes. Although a quantitative limit on the envelope contribution to the line emission requires detailed modeling for the individual sources, which will be done in a subsequent paper, a first assessment can be made with more generic models containing either only a Keplerian disk or a disk embedded in an envelope (see Appendix B). For IRAS 04302, both the $\mathrm{C}^{17} \mathrm{O}$ and $\mathrm{H}_{2} \mathrm{CO}$ visibility amplitude profiles can be reproduced without an envelope. This suggests that there is very little envelope contribution for this source, consistent with the $p v$ diagrams. A disk is also sufficient to reproduce the visibility amplitudes at velocities $>|1| \mathrm{km} \mathrm{s}^{-1}$ from the systemic velocity toward L1489, L1527, and TMC1A. For the low velocities, a small envelope contribution is required. The line emission presented here is thus dominated by the disk.

Although both the $\mathrm{C}^{17} \mathrm{O}$ and $\mathrm{H}_{2} \mathrm{CO}$ emission originates predominantly from the disk, the $\mathrm{C}^{17} \mathrm{O}$ emission extends to higher velocities than the $\mathrm{H}_{2} \mathrm{CO}$ emission in IRAS 04302, L1527, and TMC1A. This is more easily visualized in the spectra presented in Figure A2. These spectra are extracted in a $6^{\prime \prime}$ circular aperture and only include pixels with $>3 \sigma$ emission. While $\mathrm{H}_{2} \mathrm{CO}$ is brighter at intermediate velocities than $\mathrm{C}^{17} \mathrm{O}$ (even when correcting for differences in emitting area), it is not present at the highest velocities. Thus, $\mathrm{H}_{2} \mathrm{CO}$ emission seems to be absent in the inner disk in these sources, which for TMC1A is also visible in the moment zero map (Figure 1). However, in L1489, both molecules have similar maximum velocities. Toward TMC1, they extend to the same redshifted velocity, while $\mathrm{C}^{17} \mathrm{O}$ emission is strongly decreased at blueshifted velocities as compared to the redshifted velocities.

\section{2. $\mathrm{C}^{17} \mathrm{O}$ and $\mathrm{H}_{2} \mathrm{CO}$ Column Densities and Abundances}

To compare the $\mathrm{C}^{17} \mathrm{O}$ and $\mathrm{H}_{2} \mathrm{CO}$ observations between the different sources more quantitatively, we calculate diskaveraged total column densities, $N_{T}$, assuming optically thin emission in local thermodynamic equilibrium (LTE) using

$$
\frac{4 \pi F \Delta v}{A_{u l} \Omega h c g_{\text {up }}}=\frac{N_{T}}{Q\left(T_{\text {rot }}\right)} e^{-E_{\text {up }} / k T_{\text {rot }}},
$$

where $F \Delta v$ is the integrated flux density; $A_{u l}$ is the Einstein A coefficient; $\Omega$ is the solid angle subtended by the source; $E_{\text {up }}$ and $g_{\text {up }}$ are the upper-level energy and degeneracy, respectively; and $T_{\text {rot }}$ is the rotational temperature.

The integrated fluxes are measured over the dust-emitting area (Table 3). We note that this does not necessarily encompass the total line flux, but it will allow for an abundance estimate as described below. A temperature of $30 \mathrm{~K}$ is adopted for $\mathrm{C}^{17} \mathrm{O}$ and $100 \mathrm{~K}$ for $\mathrm{H}_{2} \mathrm{CO}$, as these are slightly above their freeze-out temperatures. The $\mathrm{C}^{17} \mathrm{O}$ column density ranges between $\sim 2$ and $20 \times 10^{15} \mathrm{~cm}^{-2}$, with the lowest value toward L1489 and the highest value toward TMC1A. The $\mathrm{H}_{2} \mathrm{CO}$ column density is about an order of magnitude lower, with values between $\sim 4$ and $18 \times 10^{14} \mathrm{~cm}^{-2}$. The lowest value is found toward TMC1A, and the highest value is found toward L1527. Changing the temperature for $\mathrm{H}_{2} \mathrm{CO}$ to $30 \mathrm{~K}$ decreases the column densities by only a factor of $\lesssim 3$.

The $\mathrm{H}_{2} \mathrm{CO}$ column density toward L1527 is a factor of 3-6 higher than previously derived by Sakai et al. (2014a), possibly because they integrated over different areas and velocity ranges for the envelope, disk, and envelope-disk interface. Integrating the $\mathrm{H}_{2} \mathrm{CO}$ emission over a circular aperture of $0 . ! 5$ and excluding the central $|\Delta v| \leqslant 1.0 \mathrm{~km} \mathrm{~s}^{-1}$ channels to limit the contribution from the envelope and resolved-out emission results in an $\mathrm{H}_{2} \mathrm{CO}$ column density of $9.7 \times 10^{13} \mathrm{~cm}^{-2}$, only a factor of 2-3 higher than that found by Sakai et al. (2014a). 

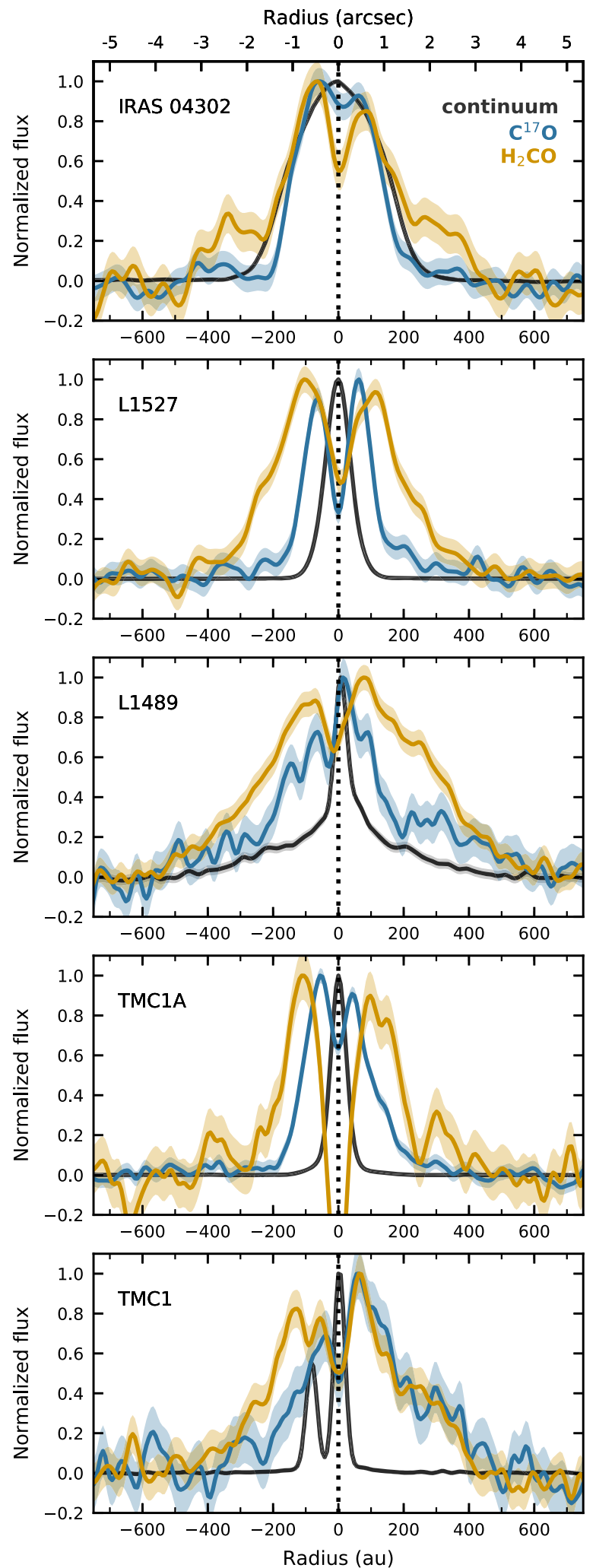

Figure 3. Normalized radial cuts along the disk major axis for the $1.3 \mathrm{~mm}$ continuum flux (black) and the $\mathrm{C}^{17} \mathrm{O}$ (blue) and $\mathrm{H}_{2} \mathrm{CO}$ (orange) integrated intensities. The shaded area shows the $3 \sigma$ uncertainty.

Pegues et al. (2020) found $\mathrm{H}_{2} \mathrm{CO}$ column densities spanning 3 orders of magnitude $\left(\sim 5 \times 10^{11}-5 \times 10^{14} \mathrm{~cm}^{-2}\right)$ for a sample of 13 Class II disks. The values derived here for Class I disks are thus similar to the high end ( $\lesssim 4$ times higher) of the values for Class II disks.
An assessment of the molecular abundances can be made by estimating the $\mathrm{H}_{2}$ column density from the continuum flux. First, we calculate the disk dust masses, $M_{\text {dust }}$, from the integrated continuum fluxes, $F_{\nu}$, using

$$
M_{\text {dust }}=\frac{D^{2} F_{\nu}}{\kappa_{\nu} B_{\nu}\left(T_{\text {dust }}\right)}
$$

where $D$ is the distance to the source, $\kappa_{\nu}$ is the dust opacity with the assumption of optically thin emission, and $B_{\nu}$ is the Planck function for a temperature $T_{\text {dust }}$ (Hildebrand 1983). Adopting a dust opacity of $\kappa_{1.3 \mathrm{~mm}}=2.25 \mathrm{~cm}^{2} \mathrm{~g}^{-1}$, as used for Class II disks by, e.g., Ansdell et al. (2016), and a dust temperature of $30 \mathrm{~K}$ similar to, e.g., Tobin et al. (2015) for embedded disks results in disk dust masses between 3.7 $M_{E}$ for TMC1-E and $75 M_{E}$ for TMC1A. Using the same dust opacity as for Class II disks is probably reasonable if grain growth starts early on in the disk formation process. However, adopting $\kappa_{1.3 \mathrm{~mm}}=0.899 \mathrm{~cm}^{2} \mathrm{~g}^{-1}$, as is often done for protostellar disks and envelopes (e.g., Jørgensen et al. 2007; Andersen et al. 2019; Tobin et al. 2020), only affects the molecular abundances by a factor of $\sim 2$. Assuming a gas-to-dust ratio of 100 and using the size of the emitting region, these dust masses result in $\mathrm{H}_{2}$ column densities of $2-90 \times 10^{23} \mathrm{~cm}^{-2}$.

The resulting $\mathrm{C}^{17} \mathrm{O}$ and $\mathrm{H}_{2} \mathrm{CO}$ abundances are listed in Table 3 . For $\mathrm{C}^{17} \mathrm{O}$, the abundances range between $1.2 \times 10^{-8}$ and $1.2 \times 10^{-7}$. Assuming a $\mathrm{C}^{16} \mathrm{O} / \mathrm{C}^{17} \mathrm{O}$ ratio of 1792 (as in the ISM; Wilson \& Rood 1994), a CO ISM abundance of $10^{-4}$ with respect to $\mathrm{H}_{2}$ corresponds to a $\mathrm{C}^{17} \mathrm{O}$ abundance of $5.6 \times 10^{-8}$. The derived $\mathrm{C}^{17} \mathrm{O}$ abundances are thus within a factor of 5 of the ISM abundance, suggesting that no substantial processing has happened, as observed for Class II disks where the $\mathrm{CO}$ abundance can be 2 orders of magnitude below the ISM value (e.g., Favre et al. 2013). These results are consistent with the results from Zhang et al. (2020) for three Class I disks in Taurus (including TMC1A) but not with the order-of-magnitude depletion found by Bergner et al. (2020) for two Class I disks in Serpens. For $\mathrm{H}_{2} \mathrm{CO}$, the abundance ranges between $\sim 3 \times 10^{-10}$ and $\sim 8 \times 10^{-9}$ in the different sources, except for TMC1A, where the abundance is $\sim 5 \times 10^{-11}$, probably due to the absence of emission in the inner region. Abundances around $10^{-10}-10^{-9}$ are consistent with chemical models for protoplanetary disks (e.g., Willacy \& Woods 2009; Walsh et al. 2014). However, $\mathrm{H}_{2} \mathrm{CO}$ abundances derived for TW Hya and HD 163296 are 2-3 orders of magnitude lower, $8.9 \times 10^{-13}$ and $6.3 \times 10^{-12}$, respectively (Carney et al. 2019).

A caveat in determining these abundances is the assumption that the continuum and line emission are optically thin. As discussed in Section 3.1, there is likely an optically thick dust component that would result in underestimates of the dust masses and overestimates of the abundances. On the other hand, optically thick dust hides molecular line emission originating below its $\tau=1$ surface, which leads to underestimates of the abundances. Based on the results from Zhang et al. (2020), $\mathrm{C}^{17} \mathrm{O}$ may be optically thick in Class I disks. This would also result in underestimating the abundances. Scaling the dust temperature used in Equation (2) with luminosity, as done by Tobin et al. (2020) for embedded disks in Orion, results in dust masses lower by a factor of $\sim 2$ and therefore slightly higher abundances. Moreover, the integrated line flux is assumed to originate solely in the disk, but as shown in Figure 4, there can be envelope emission present. Finally, the $\mathrm{H}_{2} \mathrm{CO}$ emission originates in the disk surface layers, which 
IRAS 04302
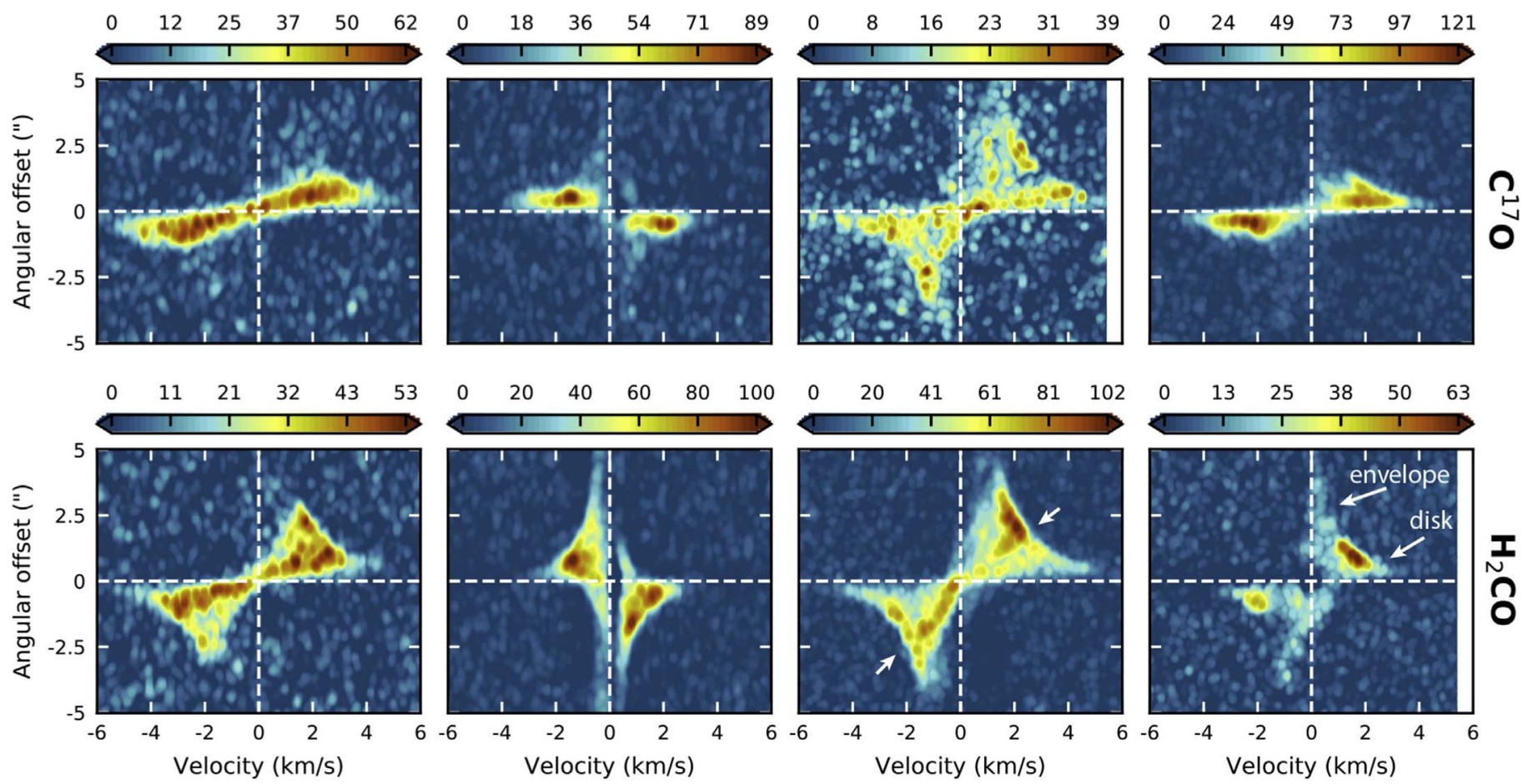

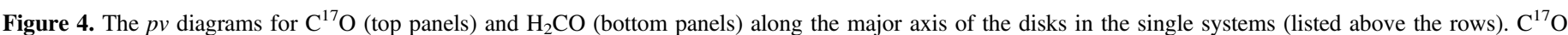

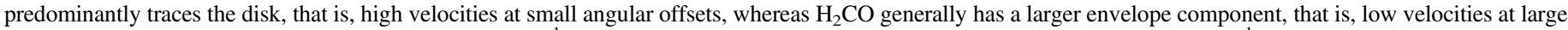

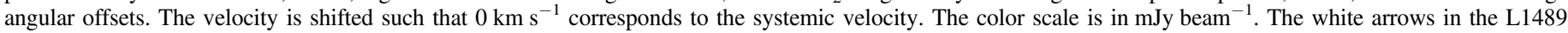
$\mathrm{H}_{2} \mathrm{CO}$ panel highlight the linear feature that is described in the text.

means the abundances are higher than derived here assuming emission originating throughout the disk. To take all these effects into account, source-specific models are required.

\section{3. $\mathrm{HDO}$ and $\mathrm{CH}_{3} \mathrm{OH}$ Upper Limits}

Water and methanol form on ice-covered dust grains and thermally desorb into the gas phase at temperatures $\sim 100-150 \mathrm{~K}$. These molecules are thus expected to trace the hot region inside the water snowline. The observations cover one HDO (deuterated water) transition $\left(3_{1,2}-2_{2,1}\right)$ with an upper-level energy of $168 \mathrm{~K}$ and 16 transitions in the $\mathrm{CH}_{3} \mathrm{OH} J=5_{k}-4_{k}$ branch with upper-level energies ranging between 34 and $131 \mathrm{~K}$. None of these lines are detected in any of the disks.

To compare these nondetections to observations in other systems, a $3 \sigma$ upper limit is calculated for the disk-averaged total column density by substituting

$$
3 \sigma=3 \times 1.1 \sqrt{\delta v \Delta V} \times \mathrm{rms}
$$

for the integrated flux density, $F \Delta v$, in Equation (1). Here $\delta v$ is the velocity resolution, and $\Delta V$ is the line width expected based on other line detections. The factor of 1.1 takes a $10 \%$ calibration uncertainty into account. Assuming the water and methanol emission arises from the innermost part of the disk, the rms is calculated from the baseline of the spectrum integrated over a central 0.5 diameter aperture ( $\sim$ one beam) and amounts to $\sim 2.7 \mathrm{mJy}$ for $\mathrm{HDO}$ and $\sim 3.0 \mathrm{mJy}$ for $\mathrm{CH}_{3} \mathrm{OH}$. A line width of $4 \mathrm{~km} \mathrm{~s}^{-1}$ and a rotational temperature of $100 \mathrm{~K}$ are adopted.

A $3 \sigma$ column density upper limit of $\sim 8 \times 10^{13} \mathrm{~cm}^{-2}$ is then found for HDO. This is 1-2 orders of magnitude below the column densities derived for the Class 0 sources NGC1333
IRAS2A, NGC1333 IRAS4A-NW, and NGC1333 IRAS4B $\left(\sim 10^{15}-10^{16} \mathrm{~cm}^{-2}\right.$; Persson et al. 2014$)$ and more than 3 orders of magnitude lower than toward the Class 0 source IRAS 16293A ( $\sim 5 \times 10^{17} \mathrm{~cm}^{-2}$; Persson et al. 2013). Taking into account the larger beam size of the earlier observations $\left(\sim 1^{\prime \prime}\right)$ lowers the column density derived here by only a factor of $\sim 4$. Furthermore, Taquet et al. (2013) showed that the HDO observations toward NGC1333 IRAS2A and NGC1333 IRAS4A are consistent with column densities up to $10^{19}$ and $10^{18} \mathrm{~cm}^{-2}$, respectively, using a grid of non-LTE large velocity gradient $(\mathrm{LVG})$ radiative transfer models.

For $\mathrm{CH}_{3} \mathrm{OH}$, the $5_{0,5}-4_{0,4}$ (A) transition provides the most stringent upper limit of $\sim 8 \times 10^{14} \mathrm{~cm}^{-2}$. This upper limit is orders of magnitude lower than the column density toward the Class 0 source IRAS $16293\left(2 \times 10^{19} \mathrm{~cm}^{-2}\right.$ within a $70 \mathrm{au}$ beam; Jørgensen et al. 2016) and the young disk around the outbursting star V883 Ori (disk-averaged column density of $\sim 1.0 \times 10^{17} \mathrm{~cm}^{-2}$; van 't Hoff et al. 2018b). A similarly low upper limit $\left(5 \times 10^{14} \mathrm{~cm}^{-2}\right)$ was found for a sample of 12 Class I disks in Ophiuchus (Artur de la Villarmois et al. 2019). However, this upper limit is not stringent enough to constrain the column down to the value observed in the TW Hya protoplanetary disk (peak column density of 3-6 $610^{12} \mathrm{~cm}^{-2}$; Walsh et al. 2016) or the upper limit in the Herbig Ae disk HD 163296 (disk-averaged upper limit of $5 \times 10^{11} \mathrm{~cm}^{-2}$; Carney et al. 2019).

For a better comparison with other sources, column density ratios are calculated with respect to $\mathrm{H}_{2}$ and $\mathrm{H}_{2} \mathrm{CO}$ and reported in Table 3. Using the $\mathrm{H}_{2}$ column density derived from the continuum flux, upper limits of $\sim 1-40 \times 10^{-10}$ are found for the HDO abundance. The $\mathrm{CH}_{3} \mathrm{OH}$ upper limits range between 1 and $40 \times 10^{-9}$. This is orders of magnitude lower than what is 
Table 3

Column Densities and Column Density Ratios

\begin{tabular}{|c|c|c|c|c|c|c|}
\hline Source & Molecule & $\begin{array}{c}\text { Area }^{\mathrm{a}} \\
(\operatorname{arcsec} \times \operatorname{arcsec})\end{array}$ & $\begin{array}{c}F_{\mathrm{int}}^{\mathrm{b}} \\
\left(\mathrm{Jy} \mathrm{km} \mathrm{s}^{-1}\right)\end{array}$ & $\begin{array}{c}N^{\mathrm{c}} \\
\left(\mathrm{cm}^{-2}\right)\end{array}$ & $N / N\left(\mathrm{H}_{2}\right)^{\mathrm{d}}$ & $N / N\left(\mathrm{H}_{2} \mathrm{CO}\right)^{c}$ \\
\hline \multirow[t]{4}{*}{ IRAS 04302} & $\mathrm{C}^{17} \mathrm{O}$ & $3.95 \times 1.01$ & $1.4 \pm 0.05$ & $5.5 \pm 0.41 \times 10^{15}$ & $3.8 \times 10^{-8}$ & 46 \\
\hline & $\mathrm{H}_{2} \mathrm{CO}$ & $3.95 \times 1.01$ & $1.5 \pm 0.05$ & $1.2 \pm 0.04 \times 10^{14}$ & $8.4 \times 10^{-10}$ & $\ldots$ \\
\hline & HDO & $0.50 \times 0.50$ & $<4.5 \times 10^{-3}$ & $<7.4 \times 10^{13}$ & $<5.3 \times 10^{-10}$ & $<0.62$ \\
\hline & $\mathrm{CH}_{3} \mathrm{OH}$ & $0.50 \times 0.50$ & $<6.9 \times 10^{-3}$ & $<7.3 \times 10^{14}$ & $<5.2 \times 10^{-9}$ & $<6.1$ \\
\hline \multirow[t]{4}{*}{ L1489 } & $\mathrm{C}^{17} \mathrm{O}$ & $4.05 \times 2.19$ & $1.5 \pm 0.11$ & $2.3 \pm 0.40 \times 10^{15}$ & $1.2 \times 10^{-7}$ & 15 \\
\hline & $\mathrm{H}_{2} \mathrm{CO}$ & $4.05 \times 2.19$ & $4.2 \pm 0.11$ & $1.5 \pm 0.04 \times 10^{14}$ & $7.6 \times 10^{-9}$ & $\cdots$ \\
\hline & HDO & $0.50 \times 0.50$ & $<5.0 \times 10^{-3}$ & $<8.3 \times 10^{13}$ & $<4.2 \times 10^{-9}$ & $<0.55$ \\
\hline & $\mathrm{CH}_{3} \mathrm{OH}$ & $0.50 \times 0.50$ & $<8.4 \times 10^{-3}$ & $<8.8 \times 10^{14}$ & $<4.4 \times 10^{-8}$ & $<5.9$ \\
\hline \multirow[t]{4}{*}{ L1527 } & $\mathrm{C}^{17} \mathrm{O}$ & $1.34 \times 0.77$ & $0.54 \pm 0.03$ & $7.7 \pm 0.96 \times 10^{15}$ & $1.2 \times 10^{-8}$ & 43 \\
\hline & $\mathrm{H}_{2} \mathrm{CO}$ & $1.34 \times 0.77$ & $0.55 \pm 0.03$ & $1.8 \pm 0.10 \times 10^{14}$ & $2.7 \times 10^{-10}$ & $\ldots$ \\
\hline & HDO & $0.50 \times 0.50$ & $<5.6 \times 10^{-3}$ & $<9.2 \times 10^{13}$ & $<1.4 \times 10^{-10}$ & $<0.51$ \\
\hline & $\mathrm{CH}_{3} \mathrm{OH}$ & $0.50 \times 0.50$ & $<7.9 \times 10^{-3}$ & $<8.3 \times 10^{14}$ & $<1.3 \times 10^{-9}$ & $<4.6$ \\
\hline \multirow[t]{4}{*}{ TMC1A } & $\mathrm{C}^{17} \mathrm{O}$ & $0.93 \times 0.88$ & $1.1 \pm 0.02$ & $2.0 \pm 0.08 \times 10^{16}$ & $2.3 \times 10^{-8}$ & 488 \\
\hline & $\mathrm{H}_{2} \mathrm{CO}$ & $0.93 \times 0.88$ & $0.10 \pm 0.02$ & $4.1 \pm 0.82 \times 10^{13}$ & $4.6 \times 10^{-11}$ & $\cdots$ \\
\hline & HDO & $0.50 \times 0.50$ & $<5.0 \times 10^{-3}$ & $<8.3 \times 10^{13}$ & $<9.3 \times 10^{-11}$ & $<2.0$ \\
\hline & $\mathrm{CH}_{3} \mathrm{OH}$ & $0.50 \times 0.50$ & $<7.7 \times 10^{-3}$ & $<8.1 \times 10^{14}$ & $<9.1 \times 10^{-10}$ & $<18$ \\
\hline \multirow[t]{4}{*}{ TMC1-E } & $\mathrm{C}^{17} \mathrm{O}$ & $0.71 \times 0.54$ & $0.10 \pm 0.01$ & $3.6 \pm 0.85 \times 10^{15}$ & $3.9 \times 10^{-8}$ & 33 \\
\hline & $\mathrm{H}_{2} \mathrm{CO}$ & $0.71 \times 0.54$ & $0.12 \pm 0.01$ & $1.1 \pm 0.09 \times 10^{14}$ & $1.2 \times 10^{-9}$ & $\ldots$ \\
\hline & HDO & $0.50 \times 0.50$ & $<5.0 \times 10^{-3}$ & $<8.3 \times 10^{13}$ & $<8.9 \times 10^{-10}$ & $<0.75$ \\
\hline & $\mathrm{CH}_{3} \mathrm{OH}$ & $0.50 \times 0.50$ & $<7.7 \times 10^{-3}$ & $<8.1 \times 10^{14}$ & $<8.7 \times 10^{-9}$ & $<7.4$ \\
\hline \multirow[t]{4}{*}{ TMC1-W } & $\mathrm{C}^{17} \mathrm{O}$ & $0.81 \times 0.63$ & $0.12 \pm 0.01$ & $3.3 \pm 0.65 \times 10^{15}$ & $2.8 \times 10^{-8}$ & 35 \\
\hline & $\mathrm{H}_{2} \mathrm{CO}$ & $0.81 \times 0.63$ & $0.15 \pm 0.01$ & $9.5 \pm 0.66 \times 10^{13}$ & $8.0 \times 10^{-9}$ & $\ldots$ \\
\hline & HDO & $0.50 \times 0.50$ & $<5.0 \times 10^{-3}$ & $<8.3 \times 10^{13}$ & $<6.9 \times 10^{-10}$ & $<0.87$ \\
\hline & $\mathrm{CH}_{3} \mathrm{OH}$ & $0.50 \times 0.50$ & $<7.7 \times 10^{-3}$ & $<8.1 \times 10^{14}$ & $<6.8 \times 10^{-9}$ & $<8.5$ \\
\hline
\end{tabular}

Notes.

a Area over which the flux is extracted.

b Integrated flux. For HDO and $\mathrm{CH}_{3} \mathrm{OH}$, this is the $3 \sigma$ upper limit to the integrated flux.

c Column density.

d Column density with respect to $\mathrm{H}_{2}$, where the $\mathrm{H}_{2}$ column density is estimated from the continuum flux and assuming a gas-to-dust ratio of 100 .

e Column density with respect to $\mathrm{H}_{2} \mathrm{CO}$.

expected from ice observations $\left(10^{-6}-10^{-5}\right.$; Boogert et al. 2015), and thus from thermal desorption, as observed in IRAS $16293\left(\lesssim 3 \times 10^{-6}\right.$; Jørgensen et al. 2016) and V883 Ori $\left(\sim 4 \times 10^{-7}\right.$; van 't Hoff et al. 2018b). Abundances for nonthermally desorbed $\mathrm{CH}_{3} \mathrm{OH}$ in TW Hya are estimated to be $\sim 10^{-12}-10^{-11}$ (Walsh et al. 2016). Sakai et al. (2014a) detected faint $\mathrm{CH}_{3} \mathrm{OH}$ emission (from different transitions than targeted here) toward $\mathrm{L} 1527$, with a $\mathrm{CH}_{3} \mathrm{OH} / \mathrm{H}_{2} \mathrm{CO}$ ratio between 0.6 and 5.1. Our upper limit of 4.6 for $\mathrm{L} 1527$ is consistent with these values. $\mathrm{CH}_{3} \mathrm{OH} / \mathrm{H}_{2} \mathrm{CO}$ ratios of 1.3 and $<0.2$ were derived for TW Hya and HD 163296, respectively, but our $\mathrm{CH}_{3} \mathrm{OH}$ upper limit is not stringent enough to make a meaningful comparison. An assumption here is that the emitting regions of $\mathrm{CH}_{3} \mathrm{OH}$ and $\mathrm{H}_{2} \mathrm{CO}$ are cospatial. As noted in Section 3.1, $\mathrm{H}_{2} \mathrm{CO}$ seems absent in the inner disk where $\mathrm{CH}_{2} \mathrm{OH}$ is expected.

\section{Analysis}

\subsection{Temperature Structure in Edge-on Disks}

For (near) edge-on disks, CO freeze-out should be readily observable, as $\mathrm{CO}$ emission will be missing from the outer disk midplane (Dutrey et al. 2017; van 't Hoff et al. 2018a). Van 't Hoff et al. (2018a) studied the effect of CO freeze-out on the optically thick ${ }^{13} \mathrm{CO}$ and $\mathrm{C}^{18} \mathrm{O}$ emission in L1527. The less abundant $\mathrm{C}^{17} \mathrm{O}$ is expected to be optically thin and mainly traces the disk. Here we employ the models from van 't Hoff et al. (2018a) to predict the $\mathrm{C}^{17} \mathrm{O}$ emission pattern for varying degrees of $\mathrm{CO}$ freeze-out (see Figure C1): a "warm" model (no CO freeze-out), an "intermediate" model (CO freeze-out in the outer disk midplane), and a "cold" model (CO freeze-out in most of the disk, except the inner part and surface layers). Briefly, in these models, gaseous $\mathrm{CO}$ is present at a constant abundance of $10^{-4}$ with respect to $\mathrm{H}_{2}$ in the regions in the disk where $T>20 \mathrm{~K}$ and in the envelope. For the warm model, the L1527 temperature structure from Tobin et al. (2013) is adopted, and for the intermediate and cold models, the temperature is reduced by $40 \%$ and $60 \%$, respectively. There is no CO freeze-out in the 125 au disk in the warm model, while the intermediate and cold models have the $\mathrm{CO}$ snowline at 71 and $23 \mathrm{au}$, respectively. Synthetic image cubes are generated using the radiative transfer code LIME (Brinch \& Hogerheijde 2010), making use of the $\mathrm{C}^{17} \mathrm{O}$ LAMDA file (Schöier et al. 2005) for the LTE calculation, and are convolved with the observed beam size.

Figure 5 shows moment zero maps integrated over the low, intermediate, and high velocities for the warm and cold edge-on disk model. Models with and without an envelope are presented. The difference between the warm and cold model is most clearly distinguishable at intermediate velocities (Figure 5, middle row). In the absence of an envelope, the emission becomes V-shaped in the cold model, tracing the warm surface layers where $\mathrm{CO}$ is not frozen out. This V shape is not visible when there is a significant envelope contribution. The cold model differs from the warm model in that the envelope emission becomes comparable in strength to the disk emission when $\mathrm{CO}$ is frozen out in most of the disk. In the warm case, the disk emission dominates over the envelope emission. At low velocities (Figure 5, top row), the 
Warm model with envelope

$\begin{array}{llllll}0 & 16 & 31 & 47 & 62 & 78\end{array}$

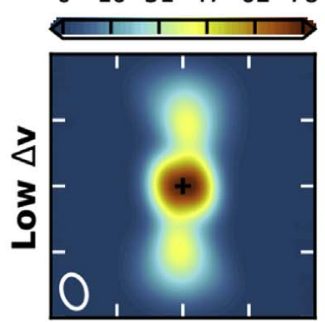

$\begin{array}{llllll}0 & 28 & 56 & 84 & 112140\end{array}$

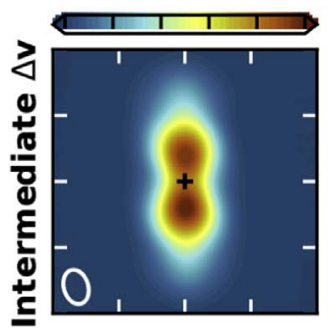

$\begin{array}{llllll}0 & 24 & 49 & 73 & 97 & 122\end{array}$

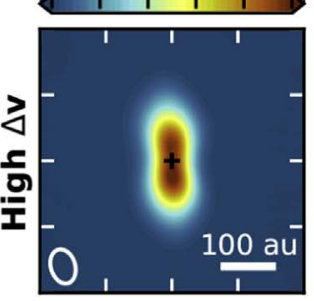

no envelope

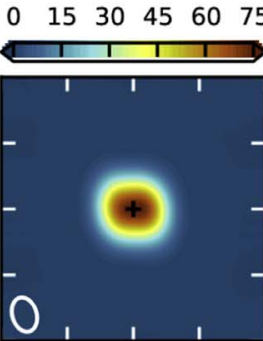

$\begin{array}{llllll}0 & 22 & 44 & 66 & 88 & 111\end{array}$
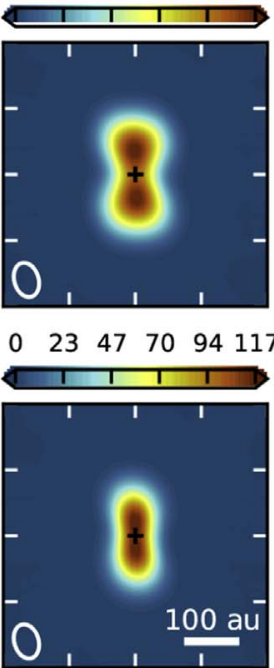

Observations

L1527

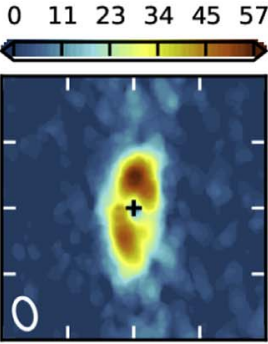

$\begin{array}{llllll}0 & 20 & 39 & 59 & 79 & 98\end{array}$
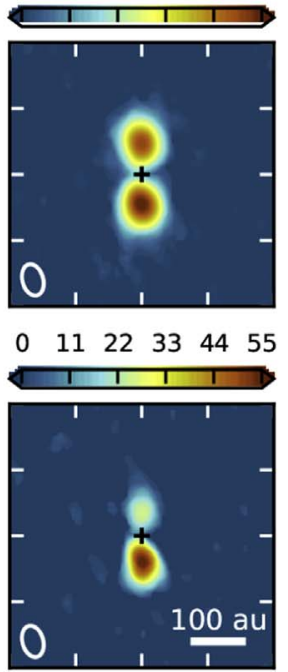

Cold model with envelope no envelope
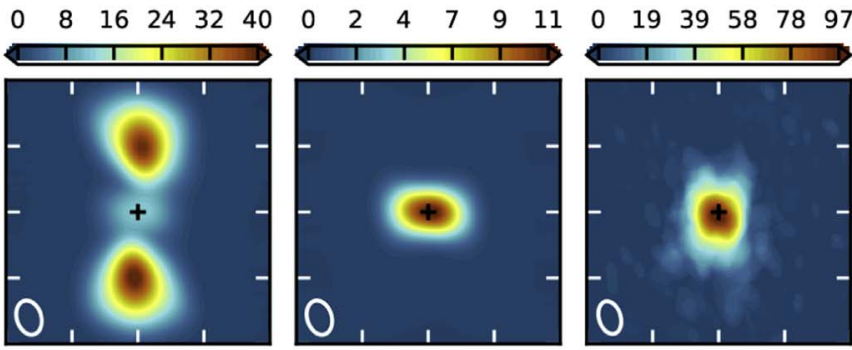

$\begin{array}{llllll}0 & 11 & 22 & 33 & 44 & 55\end{array}$
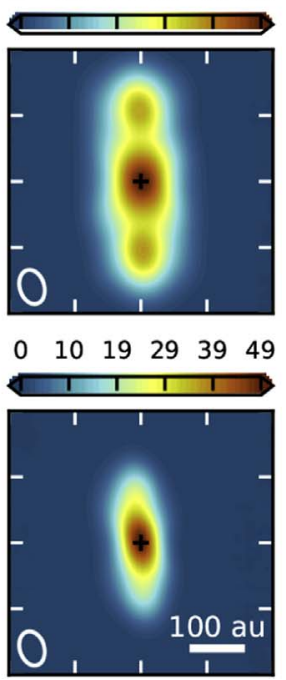

Observations

IRAS 04302
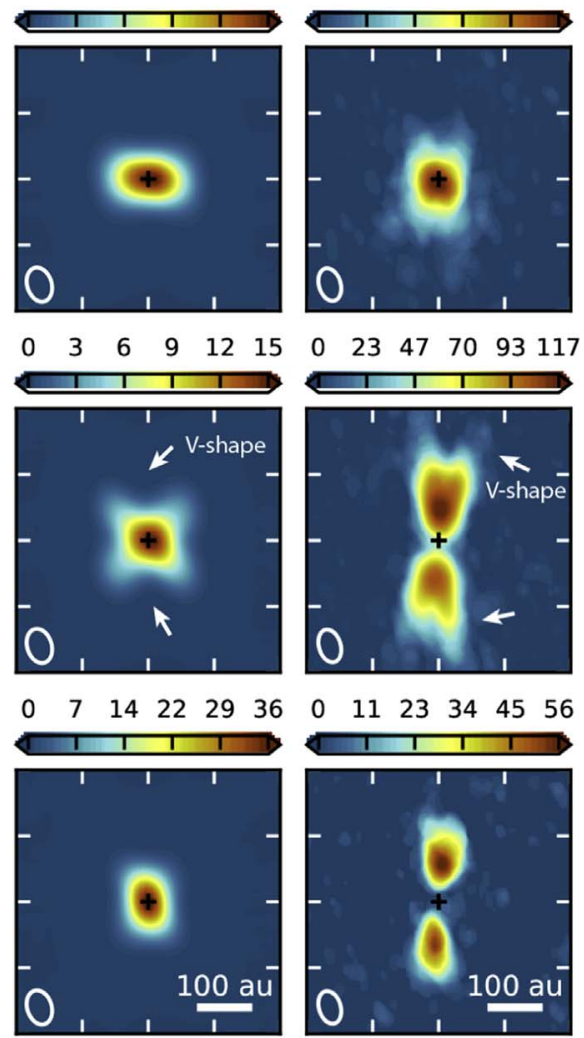

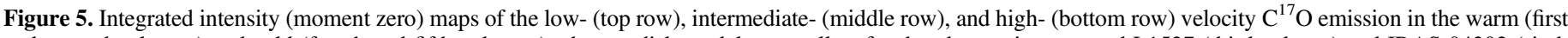

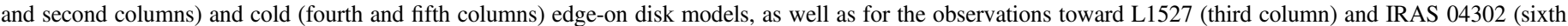

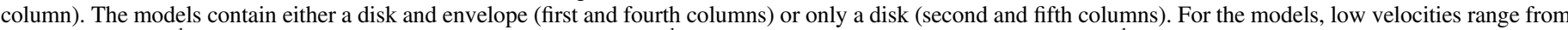

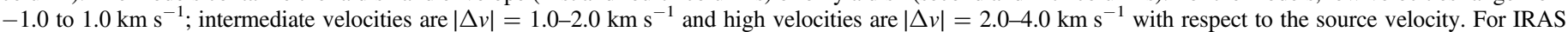

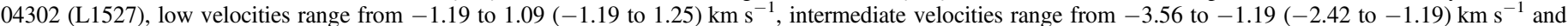

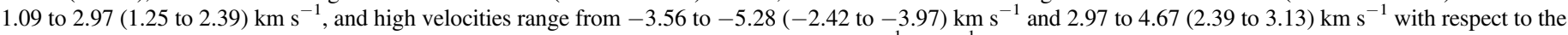

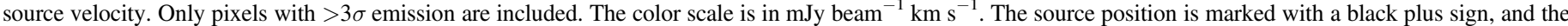

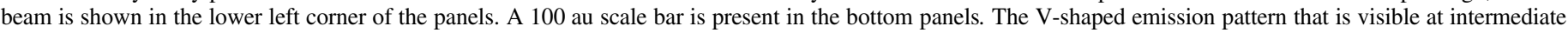
velocities in the cold model and the IRAS 04302 observations is indicated by white arrows.

difference between a warm and cold disk can be distinguished as well when an envelope is present, although in practice, this will be much harder due to resolved-out emission at these central velocities. Without an envelope, the low-velocity emission originates near the source center due to the rotation, and the models are indistinguishable, except for differences in the flux. Due to the rotation, the emission at these velocities gets projected along the minor axis of the disk (that is, east-west). At the highest velocities (Figure 5, top row), the emission originates in the inner disk, north and south of the source. If $\mathrm{CO}$ is absent in the midplane, very high angular resolution is required to observe this directly through a V-shaped pattern.

The $\mathrm{C}^{17} \mathrm{O}$ moment zero maps integrated over different velocity intervals for IRAS 04302 and L1527 are presented in Figure 5. The observations show no sign of CO freeze-out in L1527 and resemble the warm model (most clearly seen at intermediate velocities), consistent with previous results for $\mathrm{C}^{18} \mathrm{O}$ and ${ }^{13} \mathrm{CO}$ (van 't Hoff et al. 2018a). On the other hand, IRAS 04302 displays a distinct $\mathrm{V}$-shaped pattern at intermediate velocities, suggesting that $\mathrm{CO}$ is frozen out in the outer part of this much larger disk ( $\sim 250$ au, compared to 75-125 au for L1527; Tobin et al. 2013; Aso et al. 2017; Sheehan \& Eisner 2017).
The vertical distribution of the emission in both disks is highlighted in Figure 6 with vertical cuts at different radii. In L1527, the $\mathrm{C}^{17} \mathrm{O}$ emission peaks at the midplane throughout the disk, while for IRAS 04302, the peaks shift to layers higher up in the disk for radii $\gtrsim 110$ au. A first estimate of the CO snowline location can be made based on the location of the $\mathrm{V}$ shape. In the cold model, the $\mathrm{CO}$ snowline is located at $23 \mathrm{au}$, but due to the size of the beam, the base of the $\mathrm{V}$ shape and the first occurrence of a double peak in the vertical cuts are at $\sim 55$ au. In IRAS 04302 , the $\mathrm{V}$ shape begins at a radius of $\sim 130$ au, so the CO snowline location is then estimated to be around $\sim 100$ au.

A clear V-shaped pattern is also visible in the $\mathrm{H}_{2} \mathrm{CO}$ integrated emission map for IRAS 04302 (Figure 1). The V shape starts at around $55 \mathrm{au}(\sim 1$ beam offset from the continuum peak). If the reduction of $\mathrm{H}_{2} \mathrm{CO}$ in the midplane is fully due to freeze-out, the snowline is then located around (or inward of) $\sim 25 \mathrm{au}$. In L1527, $\mathrm{H}_{2} \mathrm{CO}$ emission also appears to come from surface layers, except in the outer disk (see Figures 1 and 6). The cold models show that $\mathrm{CO}$ emission from the envelope becomes comparable in strength to emission from the disk if $\mathrm{CO}$ is frozen out in a large part of the disk. Given that the envelope contribution is much larger in L1527 than in IRAS 04302, the emission peaking in the outer disk midplane is likely originating 
IRAS 04302

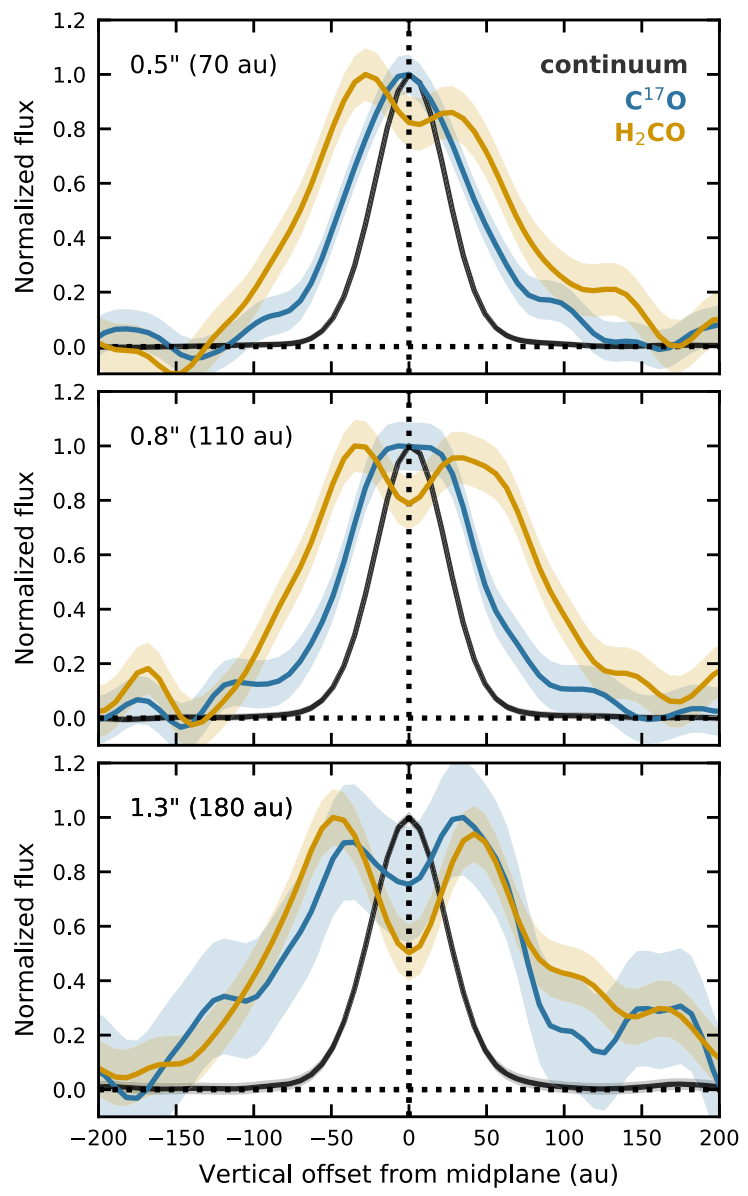

\section{$\mathbf{L 1 5 2 7}$}
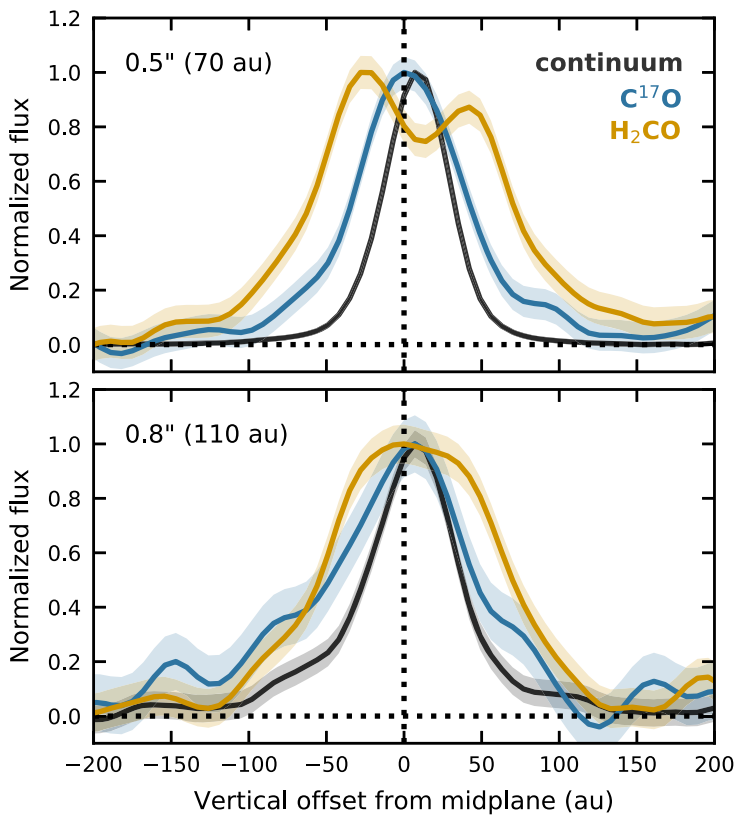

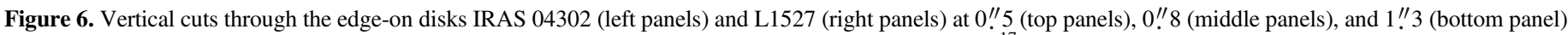

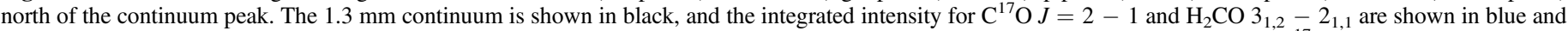

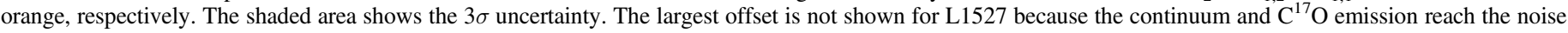
limit. The $\mathrm{H}_{2} \mathrm{CO}$ emission is single-peaked at $\sim 10$ au.

in the envelope. Instead of a clear V shape, the emission in the inner region forms two bright lanes along the continuum position. A similar pattern is seen in the individual channels. This suggests that the $\mathrm{H}_{2} \mathrm{CO}$ snowline is unresolved at the current resolution and closer in than in IRAS $04302(\lesssim 25 \mathrm{au})$.

A zeroth-order estimate of the midplane temperature profile for IRAS 04302 can be made from these two snowline estimates using a radial power law, $T \propto R^{-q}$. For disks, often a power-law exponent $q$ of 0.5 is assumed, but $q$ can range between 0.33 and 0.75 (see, e.g., Adams \& Shu 1986; Kenyon et al. 1993; Chiang \& Goldreich 1997). A power law with $q=0.75$ matches the two temperature estimates reasonably well (see Figure 7). This temperature profile is quite similar to the profile constructed for $\mathrm{L} 1527$ based on ${ }^{13} \mathrm{CO}$ and $\mathrm{C}^{18} \mathrm{O}$ temperature measurements (van 't Hoff et al. 2018a). The L1527 temperature profile predicts an $\mathrm{H}_{2} \mathrm{CO}$ snowline radius of $\lesssim 10$ au, consistent with the results derived above. Thus, IRAS 04302 is warm like L1527, with freeze-out occurring only in the outermost part of this large disk.

\subsection{Temperature Structure in Less Inclined Disks}

For less inclined disks, observing freeze-out directly is much harder; the projected area between the top and bottom layer becomes smaller (that is, the $\mathrm{V}$ shape becomes more narrow), therefore requiring higher spatial resolution to observe it. In addition, because now both the near and the far sides of the disk become visible, emission from the far side's surface layers can appear to come from the near side's midplane (see Figure C2 and Pinte et al. 2018), which makes a V shape due to emission originating only in the surface layers that are harder to observe. For the L1527 disk model, the intermediate and warm models become quite similar for an inclination of $60^{\circ}$ at this angular resolution, and only a cold disk shows a clear $\mathrm{V}$-shaped pattern (Figure 8).

Figure 9 shows the $\mathrm{C}^{17} \mathrm{O}$ moment zero maps for the intermediate inclined disks TMC1A and L1489. The disk size, stellar mass, and stellar luminosity of TMC1A are comparable to L1527. At intermediate velocities, there is no sign of a $\mathrm{V}$-shaped pattern, so these observations do not suggest substantial freeze-out of CO in TMC1A. In order to constrain the $\mathrm{CO}$ snowline a little better, models were run with snowline locations of 31, 42, and 56 au (that is, in between the cold and intermediate models). All three models show a $\mathrm{V}$ shape, suggesting that the $\mathrm{CO}$ snowline is at radii $\gtrsim 70$ au in TMC1A. This is consistent with the results from Aso et al. (2015), who found a temperature of $38 \mathrm{~K}$ at $100 \mathrm{au}$ from fitting a disk model to ALMA C ${ }^{18} \mathrm{O}$ observations, and Harsono et al. (submitted), who found a temperature of $20 \mathrm{~K}$ at $115 \mathrm{au}$. There is no sign of a $\mathrm{V}$-shaped pattern in the $\mathrm{H}_{2} \mathrm{CO}$ emission.

For L1489, the intermediate velocities show a more complex pattern, with $\mathrm{CO}$ peaking close to the source and at larger offsets 

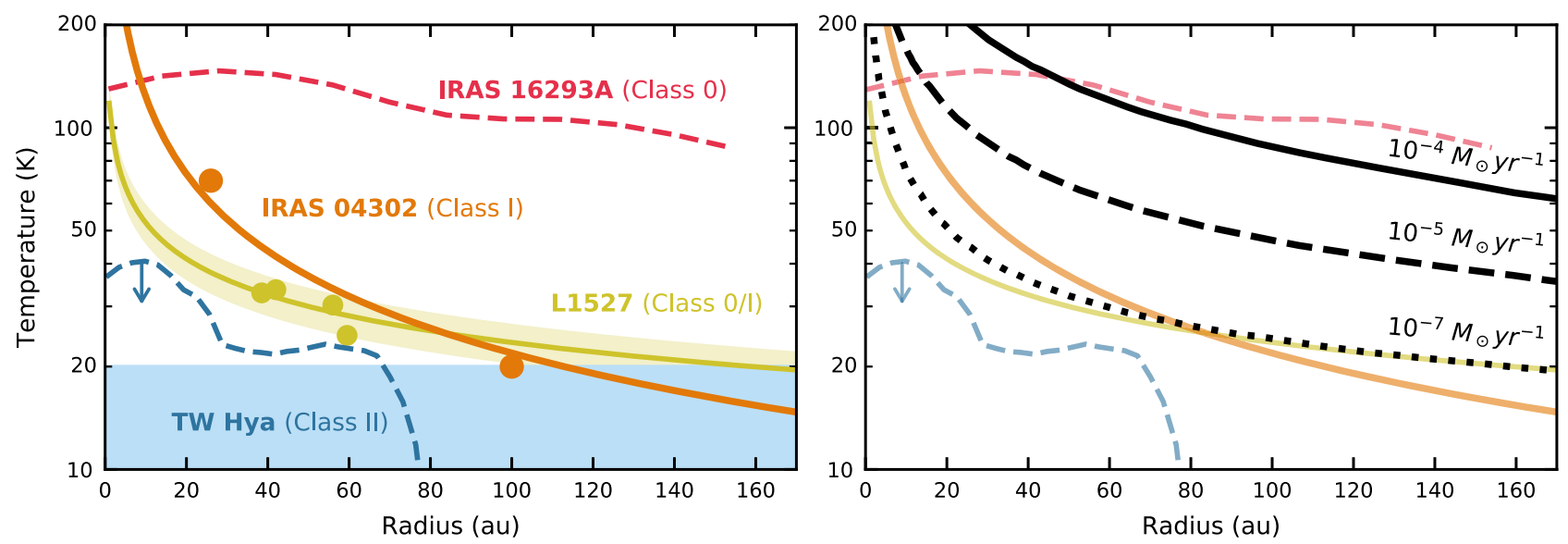

Figure 7. Left panel: radial midplane temperature profile for IRAS 04302 inferred from the $\mathrm{CO}$ and $\mathrm{H}_{2} \mathrm{CO}$ snowline estimates (orange circles). The solid orange line is a power law of the shape $T \propto R^{-0.75}$. For comparison, the temperature measurements for $\mathrm{L} 1527$ from ${ }^{13} \mathrm{CO}$ and $\mathrm{C}^{18} \mathrm{O}$ emission (yellow circles) and a power-law temperature profile with $T \propto R^{-0.35}$ (yellow line; with $1 \sigma$ uncertainty) are shown (van 't Hoff et al. 2018a), as well as the temperature profiles derived for the disklike structure in the Class 0 source IRAS 16293A (dashed red line; van 't Hoff et al. 2020) and the Class II disk TW Hya (dashed blue line; Schwarz et al. 2016). The TW Hya temperature profile traces a warmer layer above the midplane, and the midplane CO snowline is located at 20 au (e.g., van 't Hoff et al. 2017; Zhang et al. 2017). The blue shaded area denotes the temperatures at which $\mathrm{CO}$ is frozen out. Right panel: temperature profiles from the left panel overlaid with temperature profiles from embedded disk models from Harsono et al. (2015). All three models have a stellar luminosity of $1 L_{\odot}$, an envelope mass of $1 M_{\odot}$, a disk mass of $0.05 M_{\odot}$, and a disk radius of 200 au but different accretion rates of $10^{-4}$ (solid black line), $10^{-5}$ (dashed black line), and $10^{-7}$ (dotted black line) $M_{\odot} \mathrm{yr}^{-1}$ and therefore different total luminosities.

$\left(\gtrsim 2^{\prime \prime}\right)$. A similar structure was seen in $\mathrm{C}^{18} \mathrm{O}$ (Sai et al. 2020). This could be the result of nonthermal desorption of $\mathrm{CO}$ ice in the outer disk if the dust column is low enough for UV photons to penetrate (Cleeves 2016) or due to a radial temperature inversion resulting from radial drift and dust settling (Facchini et al. 2017). Such a double CO snowline has been observed for the protoplanetary disk IM Lup (Öberg et al. 2015; Cleeves 2016). The structure of the continuum emission, a bright central part and a fainter outer part, makes these plausible ideas. Another possibility is that the extended emission is due to a warm inner envelope component. The UV irradiated mass of L1489 derived from ${ }^{13} \mathrm{CO} 6-5$ emission is similar to that of L1527 and higher than for TMC1A and TMC1 (Ylldiz et al. 2015). This may provide a sufficient column along the outflow cavity wall for $\mathrm{C}^{17} \mathrm{O}$ emission to be observed. A high level of UV radiation is supported by $\mathrm{O}$ and $\mathrm{H}_{2} \mathrm{O}$ line fluxes (Karska et al. 2018).

If the edge of the compact $\mathrm{CO}$ emission is due to freeze-out, the CO snowline is located at roughly $200 \mathrm{au}$. Models based on the continuum emission have temperatures of $\sim 30$ or $\sim 20-30 \mathrm{~K}$ at $200 \mathrm{au}$ (Brinch et al. 2007 and Sai et al. 2020, respectively), so $\mathrm{CO}$ could indeed be frozen out in this region. The $\mathrm{H}_{2} \mathrm{CO}$ emission does not show a gap at $200 \mathrm{au}$, which could mean that the emission is coming from the surface layers. The $\mathrm{C}^{17} \mathrm{O}$ (and $\mathrm{C}^{18} \mathrm{O}$ ) abundance in these warmer surface layers may then be too low to be detected at the sensitivity of these observations.

\section{Discussion}

\subsection{Temperature Structure of Young Disks}

We have used observations of $\mathrm{C}^{17} \mathrm{O}$ and $\mathrm{H}_{2} \mathrm{CO}$ toward five class I disks in Taurus to address whether embedded disks are warmer than more evolved Class II disks. While the $\mathrm{C}^{17} \mathrm{O}$ observations can indicate the presence or absence of $\lesssim 20 \mathrm{~K}$ gas, the addition of $\mathrm{H}_{2} \mathrm{CO}$ observations allows one to further constrain the temperature profile. The picture that is emerging suggests that these young disks have midplanes with temperatures between $\sim 20$ and $\sim 70 \mathrm{~K}$ : cold enough for $\mathrm{H}_{2} \mathrm{CO}$ to freeze out but warm enough to retain $\mathrm{CO}$ in the gas phase (Figure 10). This suggests that, for example, the elemental $\mathrm{C} / \mathrm{O}$ ratio in both the gas and ice could be different from that in protoplanetary disks. If planet formation starts during the embedded phase, the conditions for the first steps of grain growth are then different than generally assumed.

Young disks being warmer than protoplanetary disks can also have consequences for the derived disk masses from continuum fluxes. This has been taken into consideration in recent literature by adopting a dust temperature of $30 \mathrm{~K}$ for solar-luminosity protostars (Tobin et al. 2015, 2016b; Tychoniec et al. 2018; Tychoniec et al. 2020), although not uniformly (e.g., Andersen et al. 2019; Williams et al. 2019), while $20 \mathrm{~K}$ is generally assumed for protoplanetary disks (e.g., Ansdell et al. 2016). In their study of Orion protostars, Tobin et al. (2020) took this one step further by scaling the temperature by luminosity based on a grid of radiative transfer models resulting in an average temperature of $43 \mathrm{~K}$ for a $1 L_{\odot}$ protostar. Since higher temperatures will result in lower masses for a certain continuum flux, detailed knowledge of the average disk temperature is crucial to determine the mass reservoir available for planet formation. While the current study shows that embedded disks are warmer than protoplanetary disks, and the radial temperature profiles for L1527 and IRAS 04302 hint that $30 \mathrm{~K}$ may be to low for the average disk temperature, source-specific modeling of the continuum and molecular line emission is required to address what would be an appropriate temperature to adopt for the mass derivation. However, an increase in temperature by a factor of 2 will lower the mass by only a factor of 2 (see Equation (2)), and Tobin et al. (2020) still found embedded disks to be more massive than protoplanetary disks by a factor $>4$. Differences in temperature can thus not account for the mass difference observed between embedded and protoplanetary disks.

\subsubsection{The Textbook Example of IRAS 04302}

The $\mathrm{C}^{17} \mathrm{O}$ and $\mathrm{H}_{2} \mathrm{CO}$ emission toward IRAS 04302 presents a textbook example of what one would expect to observe for an edge-on disk, that is, a direct view of the vertical structure. The $\mathrm{C}^{17} \mathrm{O}$ emission is confined to the midplane, while $\mathrm{H}_{2} \mathrm{CO}$ is tracing the surface layers. Assuming the absence of $\mathrm{H}_{2} \mathrm{CO}$ 


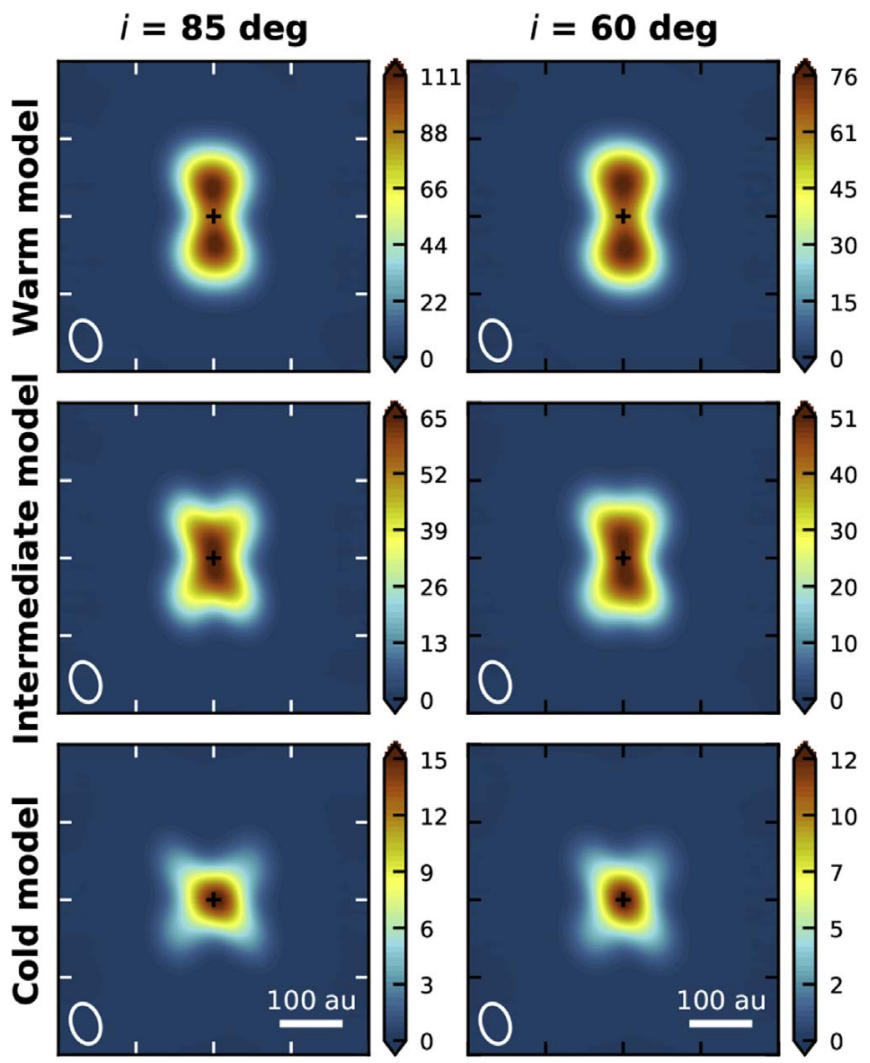

Figure 8. Integrated intensity (moment zero) maps of the intermediate-velocity $\mathrm{C}^{17} \mathrm{O} J=2-1$ emission in the warm (top row), intermediate (middle row), and cold (bottom row) disk models. The left column shows a near-edge-on disk $\left(i=85^{\circ}\right)$, as in Figure 5, and the right column shows a less inclined disk $\left(i=60^{\circ}\right)$. The velocity range $\Delta v$ is $1.0-1.9 \mathrm{~km} \mathrm{~s}^{-1}$ for $i=85^{\circ}$ and $1.3-1.8 \mathrm{~km} \mathrm{~s}^{-1}$ for $i=60^{\circ}$. The color scale is in $\mathrm{mJy}$ beam ${ }^{-1} \mathrm{~km} \mathrm{~s}^{-1}$. The source position is marked with a black plus sign, and the beam is shown in the lower left corner of the panels. A 100 au scale bar is present in the bottom panels.

emission in the midplane is due to freeze-out, we can make a first estimate not only of the radial temperature profile but also of the vertical temperature structure. At the current spatial resolution, the vertical structure is spatially resolved for radii $\gtrsim 70 \mathrm{au}$, that is, $\sim$ three beams across the disk height. At these radii, the $\mathrm{H}_{2} \mathrm{CO}$ emission peaks $\sim 30-50$ au above the midplane (at radii of 70 and $180 \mathrm{au}$, respectively), suggesting that the temperature is between $\sim 20$ and $70 \mathrm{~K}$ in the $\sim 30$ au above the midplane.

The temperature structure can be further constrained by observing molecules with a freeze-out temperature between that of $\mathrm{CO}$ and $\mathrm{H}_{2} \mathrm{CO}$, that is, between $\sim 20$ and $70 \mathrm{~K}$. Based on the UMIST database for astrochemistry (McElroy et al. 2013), examples of such molecules are $\mathrm{CN}, \mathrm{CS}, \mathrm{HCN}, \mathrm{C}_{2} \mathrm{H}, \mathrm{SO}$, and $\mathrm{H}_{2} \mathrm{CS}$ (in increasing order of freeze-out temperature). Another option would be to observe several $\mathrm{H}_{2} \mathrm{CO}$ lines because their line ratios are a good indicator of the temperature (e.g., Mangum \& Wootten 1993). These observations thus confirm that edge-on disks are well suited to study the disk vertical structure through molecular line observations.

\subsubsection{Comparison with Protostellar Envelopes and Protoplanetary Disks}

No sign of $\mathrm{CO}$ freeze-out is detected in the $\mathrm{C}^{17} \mathrm{O}$ observations of $\mathrm{L} 1527$, and while freeze-out is much more difficult to see in
L1489
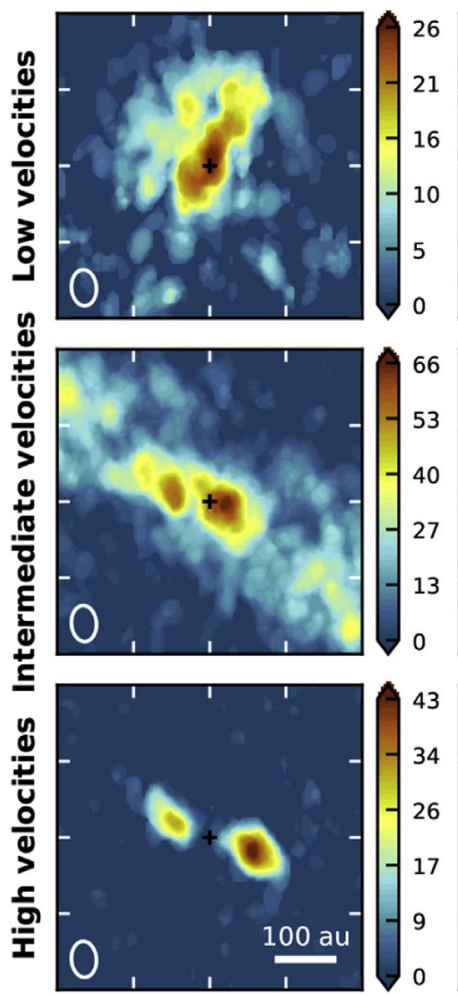

TMC1A

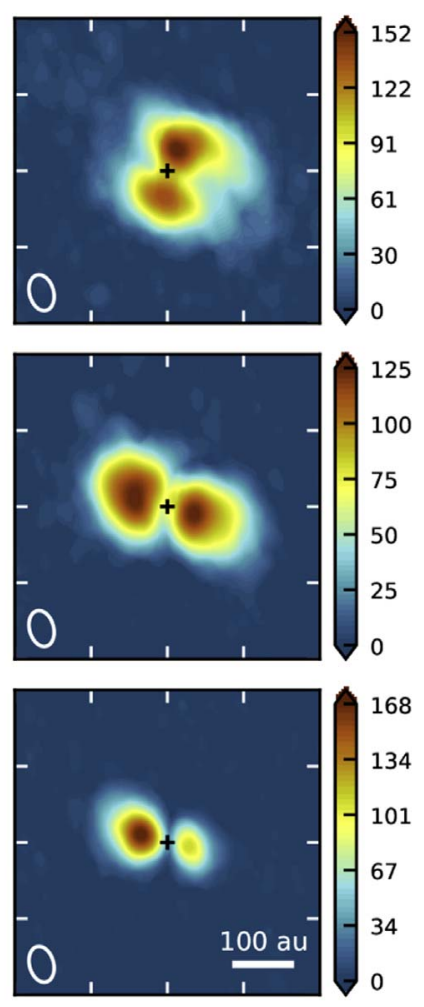

Figure 9. Integrated intensity (moment zero) maps of the low- (top row), intermediate- (middle row), and high- (bottom row) velocity $\mathrm{C}^{17} \mathrm{O} J=2-1$ emission toward L1489 (left column) and TMC1A (right column). Only pixels with $>3 \sigma$ emission are included. For TMC1A (L1489), low velocities range from -1.27 to $1.26(-0.47$ to 0.43$) \mathrm{km} \mathrm{s}^{-1}$, the intermediate velocities include $|\Delta v|=1.34-2.49(0.50-3.00) \mathrm{km} \mathrm{s}^{-1}$, and the high velocities are $|\Delta v|=$ $2.57-4.94(3.05-4.65) \mathrm{km} \mathrm{s}^{-1}$ with respect to the source velocity. The color scale is in mJy beam ${ }^{-1} \mathrm{~km} \mathrm{~s}^{-1}$. The source position is marked with a black plus sign, and the beam is shown in the lower left corner of the panels. A 100 au scale bar is present in the bottom panels.

non-edge-on disks, TMC1A does not show hints of freeze-out at radii smaller than $\sim 70$ au. A first estimate puts the $\mathrm{CO}$ snowline at $\sim 100$ au in IRAS 04302, and the CO snowline may be located around $\sim 200$ au in L1489. These young disks are thus warmer than T Tauri disks, where the snowline is typically at a few tens of $\mathrm{au}$, as can be seen in Figure 11. We only include class II disks for which a $\mathrm{CO}$ snowline location has been reported based on molecular line observations, either ${ }^{13} \mathrm{C}^{18} \mathrm{O}$ (for TW Hya; Zhang et al. 2017) or $\mathrm{N}_{2} \mathrm{H}^{+}$(Qi et al. 2019). There is no clear trend between $\mathrm{CO}$ snowline location and bolometric luminosity for either Class, but the Class I disks have $\mathrm{CO}$ snow lines at larger radii compared to Class II disks with similar bolometric luminosities.

In protostellar envelopes, snowline radii larger than expected based on the luminosity have been interpreted as a sign of a recent accretion burst (Jørgensen et al. 2015; Frimann et al. 2017; Hsieh et al. 2019). During such a time period of increased accretion, the circumstellar material heats up, shifting the snow lines outward. Once the protostar returns to its quiescent stage, the temperature adopts almost instantaneously, while the chemistry takes longer to react. During this phase, the snow lines are at larger radii than expected from the luminosity. The results in Figure 11 could thus indicate that small accretion bursts have occurred in the Class I systems and that the $\mathrm{CO}$ snow lines have not yet shifted back to their quiescent location. When such a burst should have happened 


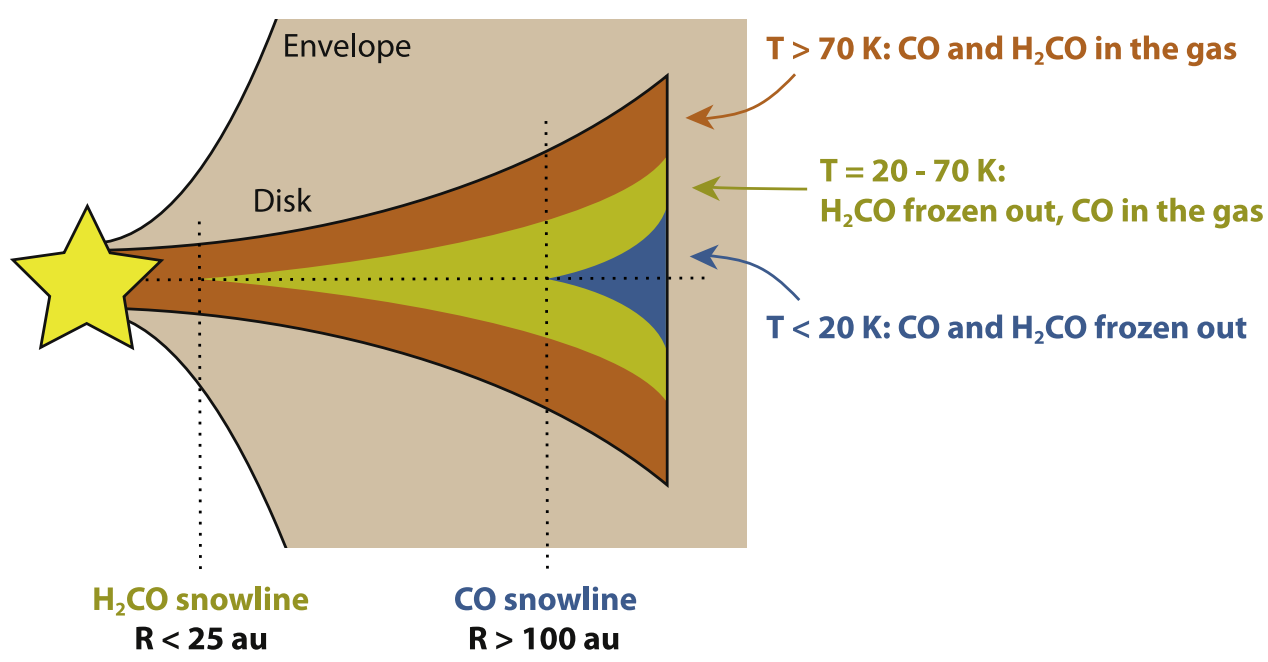

Figure 10. Schematic representation of the temperature structure derived for Class I disks based on $\mathrm{C}^{17} \mathrm{O}$ and $\mathrm{H}_{2} \mathrm{CO}$ observations. A large part of the disk midplane, or even the entire midplane, is too warm for $\mathrm{CO}$ to freeze out, unlike protoplanetary disks that have the $\mathrm{CO}$ snowline at a few tens of au. The majority of the midplane has a temperature between $\sim 20$ and $70 \mathrm{~K}$ such that $\mathrm{CO}$ is in the gas phase while $\mathrm{H}_{2} \mathrm{CO}$ is frozen out. The $\mathrm{C}^{17} \mathrm{O}$ emission therefore arises predominantly from the midplane region (yellow area), and the $\mathrm{H}_{2} \mathrm{CO}$ emission arises from the surface layers (orange region).

depends on the freeze-out timescale, $\tau_{\text {fr }}$,

$$
\tau_{\mathrm{fr}}=1 \times 10^{4} \mathrm{yr} \sqrt{\frac{10 \mathrm{~K}}{T_{\mathrm{fr}}}} \frac{10^{6} \mathrm{~cm}^{-3}}{n_{\mathrm{H}_{2}}},
$$

where $T_{\mathrm{fr}}$ is the freeze-out temperature and $n_{\mathrm{H}_{2}}$ is the gas density (Visser et al. 2012). For densities $\gtrsim 10^{8} \mathrm{~cm}^{-3}$, the CO freeze-out timescale is $\lesssim 100 \mathrm{yr}$. This could suggest that Class I protostars frequently undergo small accretion bursts. Alternatively, these young disks may have lower densities than more evolved disks. As shown by the model results from N. M. Murillo et al. (2020, in preparation), decreasing the density while keeping the luminosity constant shifts the snow lines outward. If this is what is causing the results in Figure 11, this means that embedded disks not only have different temperature structures from protoplanetary disks but also have different density structures. However, the larger disk masses derived for embedded disks compared to protoplanetary disks for similar disk radii make this unlikely (Tobin et al. 2020).

Another comparison is made in Figure 7, where the radial temperature profiles inferred for L1527 and IRAS 04302 are shown together with those for the younger Class 0 disklike structure around IRAS 16293A (van 't Hoff et al. 2020) and the Class II disk TW Hya (Schwarz et al. 2016). The young disks are warmer than the more evolved Class II disk but much colder than the Class 0 system IRAS 16293A. When making this comparison, one should keep in mind that IRAS 16293A reflects an envelope where the temperature will be larger at larger scales because of the spherical rather than disk structure. In a disk, the temperature will drop more rapidly in the radial direction due to the higher extinction compared to an envelope. Nevertheless, such an evolutionary trend is expected because the accretion rate decreases as the envelope and disk dissipate. As a consequence, heating due to viscous accretion diminishes, and hence the temperature drops, as shown by two-dimensional physical and radiative transfer models for embedded protostars (D'Alessio et al. 1997; Harsono et al. 2015). In addition, the blanketing effect of the envelope decreases as the envelope dissipates (Whitney et al. 2003).

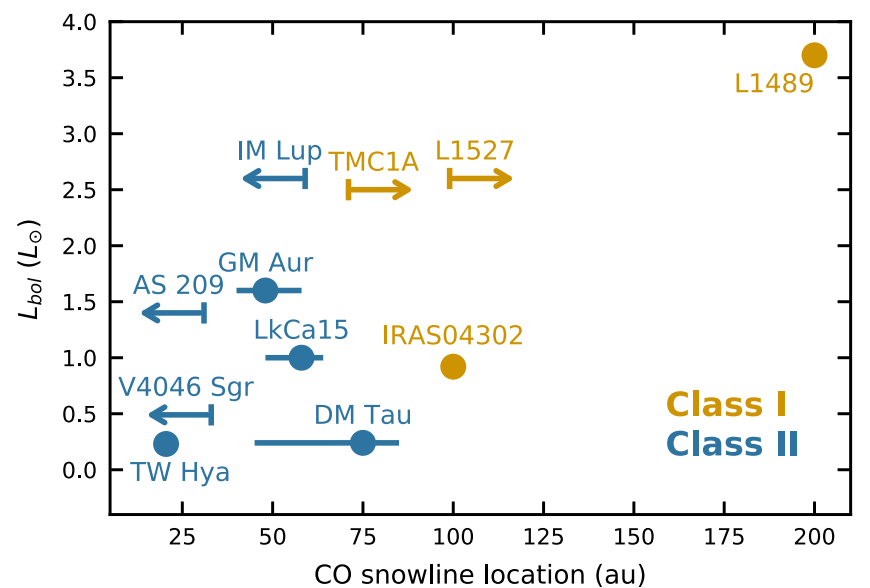

Figure 11. Overview of $\mathrm{CO}$ snowline locations in disks derived from molecular line observations as a function of bolometric luminosity. The locations for Class I disks (orange) are derived in this work using the $\mathrm{C}^{17} \mathrm{O}$ emission. Class II T Tauri disks are shown in blue. For TW Hya, the CO snowline location is determined from ${ }^{13} \mathrm{C}^{18} \mathrm{O}$ emission by Zhang et al. (2017). For the other Class II disks, the $\mathrm{CO}$ snowline is derived from $\mathrm{N}_{2} \mathrm{H}^{+}$emission by Qi et al. (2019). Arrows denote upper and lower limits.

As a first comparison between the observations and model predictions, models from Harsono et al. (2015) are overlaid on the observationally inferred temperature profiles in Figure 7 (right panel). In these models, the dust temperature is determined based on stellar irradiation and viscous accretion. Models are shown for a stellar luminosity of $1 L_{\odot}$, an envelope mass of $1 M_{\odot}$, a disk mass of $0.05 M_{\odot}$, a disk radius of $200 \mathrm{au}$, and different accretion rates. The disk mass has a negligible effect on the temperature profiles (see Harsono et al. 2015 for details). The observations for IRAS 16293A match reasonably well with the temperature profile for a heavily accreting system $\left(10^{-4} M_{\odot}\right.$ $\mathrm{yr}^{-1}$ ), consistent with estimates of the accretion rate (e.g., $\sim 5 \times 10^{-5} M_{\odot} \mathrm{yr}^{-1}$; Schöier et al. 2002). However, because in these models, the total luminosity is based on the stellar and accretion luminosity (and a contribution from the disk), the match for IRAS 16239A with a strong accretion model may just reflect the system's bolometric luminosity of $20 L_{\odot}$. In contrast, the temperature profiles for L1527 and IRAS 04302 are comparable to the colder $10^{-7} M_{\odot} \mathrm{yr}^{-1}$ model, consistent with 
the accretion rate of $\sim 3 \times 10^{-7} M_{\odot} \mathrm{yr}^{-1}$ for L1527 (see van 't Hoff et al. 2018a). Similar accretion rates on the order of $10^{-7} M_{\odot} \mathrm{yr}^{-1}$ have been reported for L1489, TMC1A, and TMC1 (e.g., Mottram et al. 2017; Yen et al. 2017) based on the bolometric luminosities (see, e.g., Stahler et al. 1980; Palla \& Stahler 1993). We are not aware of a measurement toward IRAS 04302, but our very preliminary modeling results (M. L. R. van 't Hoff et al. 2020, in preparation) are consistent with an accretion rate on the order of $10^{-7} M_{\odot} \mathrm{yr}^{-1}$. Measured accretion rates for TW Hya range between $\sim 2 \times 10^{-10}$ and $2 \times 10^{-9} M_{\odot} \mathrm{yr}^{-1}$ (e.g., Herczeg \& Hillenbrand 2008; Curran et al. 2011; Ingleby et al. 2013), and accretion rates of $\sim 10^{-10}-10^{-8} M_{\odot} \mathrm{yr}^{-1}$ are typically measured for protoplanetary disks around $\mathrm{T}$ Tauri stars (see Hartmann et al. 2016 for a review).

The results presented here thus provide observational evidence for cooling of the circumstellar material during evolution. More sources need to be observed to confirm this trend and answer more detailed questions, such as, when has a disk cooled down sufficiently for large-scale CO freeze-out? Does this already happen before the envelope dissipates? The object IRAS 04302 is a borderline Class I/Class II object embedded in the last remnants of its envelope, but it still has a temperature profile more similar to L1527 than TW Hya. Although a caveat here may be the old age of TW Hya ( 10 Myr), this hints that disks may stay warm until the envelope has fully dissipated.

\subsection{3. $T M C 1$}

For the first time, TMC1 is resolved to be a close $(\sim 85 \mathrm{au})$ binary. A possible configuration of the system could be that TMC1-E is present in the disk of TMC1-W, as observed, for example, for L1448 IRS3B (Tobin et al. 2016a). Then TMC1-E would increase the temperature on the east side of the disk. This may be an explanation for the asymmetry in the $\mathrm{C}^{17} \mathrm{O}$ emission with the emission dimmer east of TMC1-W (see Figures 3, A1 and A2). Given the upper-level energy of $16 \mathrm{~K}$, emission from the $\mathrm{C}^{17} \mathrm{O} J=2-1$ transition will decrease with temperatures increasing above $\sim 25 \mathrm{~K}$. The weaker $\mathrm{C}^{17} \mathrm{O}$ emission may thus signal a higher temperature on the east side of the disk. However, TMC1-E does not seem to cause any disturbances in the disk, such as spiral arms, although the high inclination may make this hard to see. Another possibility could be that TMC1-E is actually in front of the disk.

\subsection{Chemical Complexity in Young Disks}

One of the major questions regarding the chemical composition of planetary material is whether it contains complex organic molecules (COMs). Due to the low temperatures in protoplanetary disks, observations of COMs are very challenging because these molecules thermally desorb at temperatures $\gtrsim 100-150 \mathrm{~K}$, that is, in the inner few au. In contrast, COMs are readily detected on disk scales in protostellar envelopes (e.g., IRAS 16293, NGC1333 IRAS2A, NGC1333 IRAS4A, and B1c; Taquet et al. 2015; Jørgensen et al. 2016; van Gelder et al. 2020) and in the young disk V883 Ori, where a luminosity outburst has heated the disk and liberated the COMs from the ice mantles (van 't Hoff et al. 2018b; Lee et al. 2019).

Although young disks seem warmer than protoplanetary disks, the $\mathrm{CH}_{3} \mathrm{OH}$ and $\mathrm{HDO}$ nondetections with upper limits orders of magnitude below the column densities observed toward Class 0 protostellar envelopes suggest that they are not warm enough to have a hot core-like region with a large gas reservoir of COMs. This is consistent with recent findings by Artur de la Villarmois et al. (2019) for a sample of Class I protostars in Ophiuchus. More stringent upper limits are required for comparison with the Class II disks TW Hya and HD 163296. However, the detection of $\mathrm{HDO}$ and $\mathrm{CH}_{3} \mathrm{OH}$ may have been hindered by optically thick dust in the inner region or the high inclinations of these sources. Modeling by N. M. Murillo et al. (2020, in preparation) shows that the water snowline is very hard to detect in near-edge-on disks. These nondetections thus do not rule out the presence of HDO and $\mathrm{CH}_{3} \mathrm{OH}$; in fact, if the region where $\mathrm{HDO}$ and $\mathrm{CH}_{3} \mathrm{OH}$ are present is much smaller than the beam, they may have higher columns than the upper limits derived here. This is corroborated by the weak detection of $\mathrm{CH}_{3} \mathrm{OH}$ in L1527 (Sakai et al. 2014a). These results thus merely show that Class I disks do not have an extended hot core-like region, making the detection of COMs just as challenging as in Class II disks.

A question related to the chemical composition is whether the disk material is directly inherited from the cloud, processed en route to the disk, or even fully reset upon entering the disk. Young disks like L1527, where no CO freeze-out is observed, suggest that no full inheritance takes place, at least not for the most volatile species like CO. Ice in the outer disk of IRAS 04302 could be inherited. However, the freeze-out timescale for densities $>10^{6} \mathrm{~cm}^{-3}$ is $<10^{4} \mathrm{yr}$, so this $\mathrm{CO}$ could have sublimated upon entering the disk and frozen out as the disk cooled (see, e.g., Visser et al. 2009). Without CO ice, additional grain-surface formation of COMs will be limited in the young disks. So if COMs are present in more evolved disks, as, for example, shown for V883 Ori, they must have been inherited from a colder precollapse phase. Physicochemical models show that prestellar methanol can indeed be incorporated into the disk (Drozdovskaya et al. 2014).

\subsection{Decrease in $\mathrm{H}_{2} \mathrm{CO}$ in the Inner Disk}

While the $\mathrm{H}_{2} \mathrm{CO}$ emission is brighter than the $\mathrm{C}^{17} \mathrm{O}$ emission at intermediate velocities, no $\mathrm{H}_{2} \mathrm{CO}$ emission is detected at the highest velocities in IRAS 04302, L1527, and TMC1A, suggesting a reduction in $\mathrm{H}_{2} \mathrm{CO}$ flux in the inner $\lesssim 20-30$ au in these disks. This is not just a sensitivity issue, as, for example, $\mathrm{C}^{17} \mathrm{O}$ and $\mathrm{H}_{2} \mathrm{CO}$ have similar strengths and emitting areas in channels around $+1.9 \mathrm{~km} \mathrm{~s}^{-1}$ with respect to the source velocity in L1527, while $3.05 \mathrm{~km} \mathrm{~s}^{-1}$ is the highest velocity observed for $\mathrm{C}^{17} \mathrm{O}$ and $2.60 \mathrm{~km} \mathrm{~s}^{-1}$ the highest velocity for $\mathrm{H}_{2} \mathrm{CO}$. The decrease in $\mathrm{H}_{2} \mathrm{CO}$ emission is also unlikely to be due to the continuum being optically thick because this would affect the $\mathrm{C}^{17} \mathrm{O}$ emission as well, unless there is significantly more $\mathrm{C}^{17} \mathrm{O}$ emission coming from layers above the dust millimeter $\tau=1$ surface than $\mathrm{H}_{2} \mathrm{CO}$ emission. Given the observed distributions, with $\mathrm{H}_{2} \mathrm{CO}$ being vertically more extended than $\mathrm{C}^{17} \mathrm{O}$, this seems not to be the case. Moreover, the drop in $\mathrm{H}_{2} \mathrm{CO}$ in TMC1A occurs much further out than where the dust becomes optically thick.

Formaldehyde rings have also been observed in the protoplanetary disks around TW Hya (Öberg et al. 2017), HD 163296 (Qi et al. 2013a; Carney et al. 2017), DM Tau (Henning \& Semenov 2008; Loomis et al. 2015), and DG Tau (Podio et al. 2019). Interestingly, a ring is only observed for the $3_{03}-2_{02}$ and $3_{12}-2_{11}$ transitions and not for the $5_{15}-4_{14}$ transition. Öberg et al. (2017) argued that the dust opacity cannot be the major contributor in TW Hya because the dust opacity should be higher at higher frequencies, thus for the $5_{15}-4_{14}$ 
transition. Instead, they suggested a warm inner component that is visible in the $5_{15}-4_{14}$ transition $\left(E_{\text {up }}=63 \mathrm{~K}\right)$ and not in the $3_{12}-2_{11}$ transition $\left(E_{\mathrm{up}}=33 \mathrm{~K}\right)$. For L1527, we observe the $3_{12}-2_{11}$ transition, and radiative transfer modeling for the L1527 warm disk model shows that both the $\mathrm{C}^{17} \mathrm{O}\left(E_{\text {up }}=33 \mathrm{~K}\right)$ and $\mathrm{H}_{2} \mathrm{CO}$ emission go down by a factor of $\sim 2$ if the temperature is increased by $80 \%$. An excitation effect thus seems unlikely, unless the $\mathrm{C}^{17} \mathrm{O}$ emission is optically thick. The latter is not expected, given that the $\mathrm{C}^{18} \mathrm{O}$ in $\mathrm{L} 1527$ is only marginally optically thick (van 't Hoff et al. 2018a). The absence of $\mathrm{H}_{2} \mathrm{CO}$ emission in the inner disk thus points to a reduced $\mathrm{H}_{2} \mathrm{CO}$ abundance. A lower total (gas + ice) $\mathrm{H}_{2} \mathrm{CO}$ abundance (more than an order of magnitude) in the inner $30 \mathrm{au}$ is seen in models by Visser et al. (2011), who studied the chemical evolution from prestellar core into disk, but these authors did not discuss the $\mathrm{H}_{2} \mathrm{CO}$ chemistry.

The $\mathrm{H}_{2} \mathrm{CO}$ abundance in the inner disk can be low if its formation is inefficient. It can form in both the gas and ice (e.g., Willacy \& Woods 2009; Walsh et al. 2014; Loomis et al. 2015). On the grain surfaces, the dominant formation route is through hydrogenation of CO (Watanabe \& Kouchi 2002; Cuppen et al. 2009; Fuchs et al. 2009). Since there seems to be no CO freezeout in these young disks, or only at radii $\gtrsim 100 \mathrm{au}, \mathrm{H}_{2} \mathrm{CO}$ is expected to form predominantly in the gas. Ring-shaped $\mathrm{H}_{2} \mathrm{CO}$ emission due to increased ice formation outside the $\mathrm{CO}$ snowline, as used to explain the ring observed in HD 163296 (Qi et al. 2013a), is thus not applicable to the disks in this sample.

In the gas, the reaction between $\mathrm{CH}_{3}$ and $\mathrm{O}$ is the most efficient way to form $\mathrm{H}_{2} \mathrm{CO}$ (e.g., Loomis et al. 2015). Therefore, a decrease in gas-phase $\mathrm{H}_{2} \mathrm{CO}$ formation would require a low abundance of either $\mathrm{CH}_{3}$ or $\mathrm{O} . \mathrm{CH}_{3}$ is efficiently produced by photodissociation of $\mathrm{CH}_{4}$ or through ion-molecule reactions. A low $\mathrm{CH}_{3}$ abundance thus necessitates the majority of carbon to be present in $\mathrm{CO}$, in combination with a low X-ray flux, as carbon can only be liberated from $\mathrm{CO}$ by X-ray-generated $\mathrm{He}^{+}$. Atomic oxygen is formed through photodissociation of $\mathrm{H}_{2} \mathrm{O}$ and $\mathrm{CO}_{2}$ or dissociation of $\mathrm{CO}$ via $\mathrm{X}$-ray-generated $\mathrm{He}^{+}$. A low atomic oxygen abundance would thus require a low UV and X-ray flux.

Besides a low formation rate, a high destruction rate would also decrease the amount of $\mathrm{H}_{2} \mathrm{CO}$. However, the destruction products have a limited chemistry, and re-creation of $\mathrm{H}_{2} \mathrm{CO}$ is the most likely outcome. Willacy \& Woods (2009) showed that a third of the ions formed by $\mathrm{H}_{2} \mathrm{CO}$ destruction through $\mathrm{HCO}^{+}$and $\mathrm{DCO}^{+}$ form $\mathrm{CO}$ instead of reforming $\mathrm{H}_{2} \mathrm{CO}$, leading to a depletion between 7 and 20 au for their disk model. However, this only reduces $\mathrm{H}_{2} \mathrm{CO}$ in the midplane, not in the surface layers. In addition, Henning \& Semenov (2008) suggested the conversion of $\mathrm{CO}$ into $\mathrm{CO}_{2}$-containing molecules and hydrocarbons that freeze out onto dust grains (see also Aikawa et al. 1999). However, the $\mathrm{C}^{17} \mathrm{O}$ observations do not suggest heavy $\mathrm{CO}$ depletion.

Another effect that could contribute is photodesorption of methanol ice that is inherited from earlier phases. Laboratory experiments have shown that methanol does not desorb intact upon vacuum ultraviolet (VUV) irradiation but rather leads to the release of smaller photofragments including $\mathrm{H}_{2} \mathrm{CO}$ (Bertin et al. 2016; Cruz-Diaz et al. 2016). This could lead to an increase of $\mathrm{H}_{2} \mathrm{CO}$ outside the region where $\mathrm{CH}_{3} \mathrm{OH}$ ice thermally desorbs ( $\sim 100-150 \mathrm{~K})$. Finally, turbulence may play a role, as models by Furuya \& Aikawa (2014) show the formation of $\mathrm{H}_{2} \mathrm{CO}$ rings when mixing is included. However, these rings are due to a decrease of $\mathrm{H}_{2} \mathrm{CO}$ inside the $\mathrm{CO}$ snowline and an increase outside this snowline, and these results may not be applicable to embedded disks without $\mathrm{CO}$ freeze-out. Observations of higher- excitation $\mathrm{H}_{2} \mathrm{CO}$ lines and chemical modeling with sourcespecific structures may provide further insights.

It is worth noting that Pegues et al. (2020) found both centrally peaked and centrally depressed $\mathrm{H}_{2} \mathrm{CO}$ emission profiles for a sample of 15 protoplanetary disks. A reduction of $\mathrm{H}_{2} \mathrm{CO}$ emission toward three out of the five disks in our sample could mean that the $\mathrm{H}_{2} \mathrm{CO}$ distribution is set during the embedded stage.

\section{Conclusions}

Temperature plays a key role in the physical and chemical evolution of circumstellar disks and therefore the outcome of planet formation. However, the temperature structure of young embedded disks, in which the first steps of planet formation take place, is poorly constrained. Our previous analysis of ${ }^{13} \mathrm{CO}$ and $\mathrm{C}^{18} \mathrm{O}$ emission in the young disk $\mathrm{L} 1527$ suggests that this disk is warm enough ( $T \gtrsim 20-25 \mathrm{~K}$ ) to prevent $\mathrm{CO}$ freeze-out (van 't Hoff et al. 2018a), in contrast to protoplanetary disks that show large cold outer regions where $\mathrm{CO}$ is frozen out. Here we present ALMA observations of $\mathrm{C}^{17} \mathrm{O}$ and $\mathrm{H}_{2} \mathrm{CO}$ and nondetections of $\mathrm{HDO}$ and $\mathrm{CH}_{3} \mathrm{OH}$ for five young disks in Taurus, including L1527. The observations of L1527 and, in particular, IRAS 04302, with $\mathrm{C}^{17} \mathrm{O}$ emission originating in the midplane and $\mathrm{H}_{2} \mathrm{CO}$ emission tracing the surface layers, highlight the potential of edge-on disks to study the disk vertical structure.

Based on the following results, we conclude that young disks are likely warmer than more evolved protoplanetary disks but not warm enough to have a large gas reservoir of complex molecules, like the young disk around the outbursting star V883 Ori.

1. The presence of $\mathrm{CO}$ freeze-out can be directly observed with $\mathrm{C}^{17} \mathrm{O}$ observations in edge-on disks. The disk around L1527 shows no sign of CO freeze-out, but IRAS 04302 has a large enough disk for the temperature to drop below the $\mathrm{CO}$ freeze-out temperature in the outermost part (radii $\gtrsim 100 \mathrm{au}$ ).

2. The $\mathrm{H}_{2} \mathrm{CO}$ emission originates primarily in the surface layers of IRAS 04302 and L1527. The snowline $(T \sim 70 \mathrm{~K})$ is estimated around (or inward of) $\sim 25 \mathrm{au}$ in IRAS 04302 and at $\lesssim 25$ au in L1527.

3. The presence of $\mathrm{CO}$ freeze-out is much more difficult to observe in non-edge-on disks, but the $\mathrm{C}^{17} \mathrm{O}$ emission in TMC1A suggest a snowline at radii $\gtrsim 70$ au. Two spatial components are seen in the $\mathrm{C}^{17} \mathrm{O}$ emission toward L1489. If the outer edge of the inner component is due to $\mathrm{CO}$ freeze-out, the snowline will be around $\sim 200$ au.

4. The CO snowline locations derived for the Class I disks are farther out than those found for Class II disks with similar bolometric luminosities.

5. The $\mathrm{HDO}$ and $\mathrm{CH}_{3} \mathrm{OH}$ nondetections with upper limits more than 2 orders of magnitude lower than those observed for hot cores in protostellar envelopes or the disk around the outbursting star V883 Ori suggest that these Class I disks do not have a large gas reservoir of COMs.

6. The inferred temperature profiles are consistent with trends found in radiative transfer models of diskenvelope systems with accretion rates decreasing from $10^{-4}$ to $10^{-7} M_{\odot} \mathrm{yr}^{-1}$.

As evidence is piling up for planet formation to start already during the embedded phase, adopting initial conditions based on the physical conditions in more evolved Class II disks seems inappropriate. Instead, planet formation may start in warmer 
IRAS 04302 L1527 L1489 TMC1A TMC1

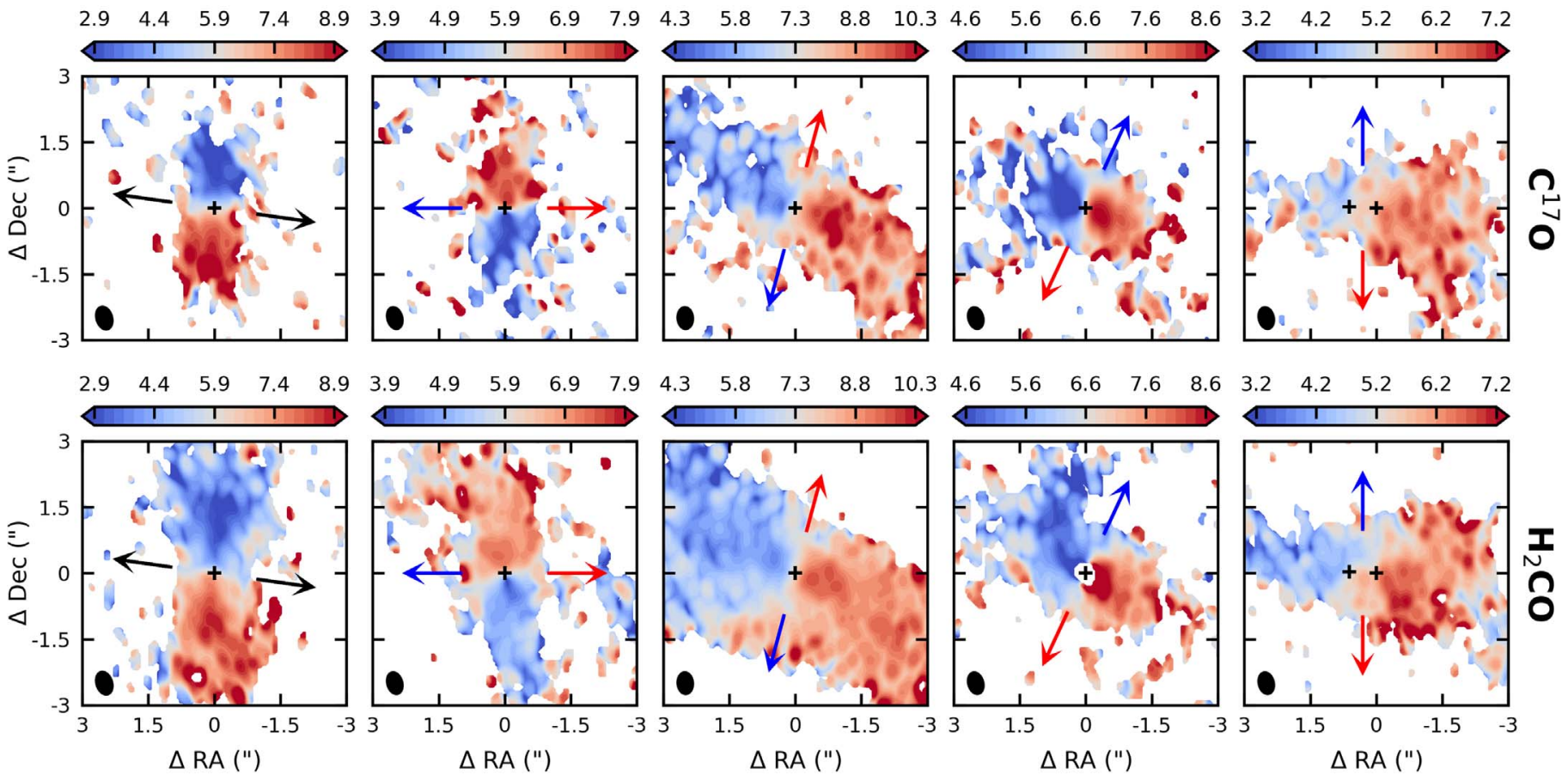

Figure A1. Moment one maps for the $\mathrm{C}^{17} \mathrm{O} J=2-1$ (top row) and $\mathrm{H}_{2} \mathrm{CO}_{1,2}-2_{1,1}$ (bottom row) transitions. The central velocity of the color scale is the systemic velocity $\left(\mathrm{km} \mathrm{s}^{-1}\right)$. The positions of the continuum peaks are marked with black plus signs, and the outflow directions are indicated by arrows. The beam is shown in the lower left corner of each panel.

conditions than generally assumed. Furthermore, without a large CO-ice reservoir, COM formation efficiency is limited in embedded disks. Observations of COMs in more evolved disks therefore suggest that these molecules are inherited from earlier phases.

We would like to thank the referee for a prompt and positive report that helped improve the paper, Patrick Sheehan for his assistance with the visibility plotting, and Gleb Fedoseev for useful discussions about the $\mathrm{H}_{2} \mathrm{CO}$ freeze-out temperature. M.L. R.H. would like to thank Yuri Aikawa for comments on an earlier version of this manuscript for her $\mathrm{PhD}$ thesis. This paper makes use of the following ALMA data: ADS/JAO. ALMA\#2017.1.01413.S. ALMA is a partnership of ESO (representing its member states), NSF (USA), and NINS (Japan), together with NRC (Canada), MOST and ASIAA (Taiwan), and KASI (Republic of Korea), in cooperation with the Republic of Chile. The Joint ALMA Observatory is operated by ESO, AUI/ NRAO, and NAOJ. Astrochemistry in Leiden is supported by the Netherlands Research School for Astronomy (NOVA). M.L. R.H. acknowledges support from a Huygens fellowship from Leiden University. J.J.T. acknowledges support from grant AST1814762 from the National Science Foundation and past support from the Homer L. Dodge Endowed Chair at the University of Oklahoma. The National Radio Astronomy Observatory is a facility of the National Science Foundation operated under cooperative agreement by Associated Universities, Inc. J.K.J. acknowledges support by the European Research Council (ERC) under the European Union's Horizon 2020 research and innovation program through ERC Consolidator Grant "S4F" (grant agreement No.646908). A.M. acknowledges funding from the European Union's Horizon 2020 research and innovation program under Marie Sklodowska-Curie grant agreement No. 823823 (RISE DUSTBUSTERS) and from the
Table A1

Overview of the Molecular Line Observations

\begin{tabular}{lcccc}
\hline \hline Molecule & Transition & $\begin{array}{c}\text { Frequency } \\
(\mathrm{GHz})\end{array}$ & $\begin{array}{c}A_{\mathrm{ul}}{ }^{\mathrm{a}} \\
\left(\mathrm{s}^{-1}\right)\end{array}$ & $\begin{array}{c}E_{\text {up }}{ }^{\mathrm{b}} \\
(\mathrm{K})\end{array}$ \\
\hline $\mathrm{C}^{17} \mathrm{O}$ & $2-1$ & 224.714385 & $6.42 \times 10^{-7}$ & 16 \\
$\mathrm{H}_{2} \mathrm{CO}$ & $3_{1,2}-2_{1,1}$ & 225.697775 & $2.77 \times 10^{-4}$ & 33 \\
$\mathrm{HDO}$ & $3_{1,2}-2_{2,1}$ & 225.896720 & $1.32 \times 10^{-5}$ & 168 \\
$\mathrm{CH}_{3} \mathrm{OH}$ & $5-4^{\mathrm{c}}$ & $241.820762^{\mathrm{d}}$ & $2-6 \times 10^{-5}$ & $34-131$ \\
\hline
\end{tabular}

Notes. Data for $\mathrm{C}^{17} \mathrm{O}$ and HDO are taken from the Jet Propulsion Laboratory Molecular Spectroscopy database (Pickett et al. 1998), and data for $\mathrm{H}_{2} \mathrm{CO}$ and $\mathrm{CH}_{3} \mathrm{OH}$ are from the Cologne Database for Molecular Spectroscopy (Müller et al. 2005).

${ }^{\mathrm{a}}$ Einstein A coefficient.

${ }^{\mathrm{b}}$ Upper-level energy.

${ }^{c}$ The spectral window covers multiple transitions in the $5_{K}-4_{K}$ branch for both A- and E-methanol (16 transitions in total).

${ }^{\mathrm{d}}$ Central frequency of the spectral window.

Deutsche Forschungsgemeinschaft (DFG; German Research Foundation), Ref. no. FOR 2634/1 ER685/11-1. C.W. acknowledges financial support from the University of Leeds and the Science and Technology Facilities Council (grant Nos. ST/R000549/1 and ST/T000287/1).

\section{Appendix A \\ Observations}

Table A1 presents an overview of the observed molecular lines. Moment one maps for $\mathrm{C}^{17} \mathrm{O}$ and $\mathrm{H}_{2} \mathrm{CO}$ toward all disks in the sample are shown in Figure $\mathrm{A} 1$, and spectra integrated over pixels with $>3 \sigma$ emission in a $6^{\prime \prime}$ circular aperture are presented in Figure A2. 

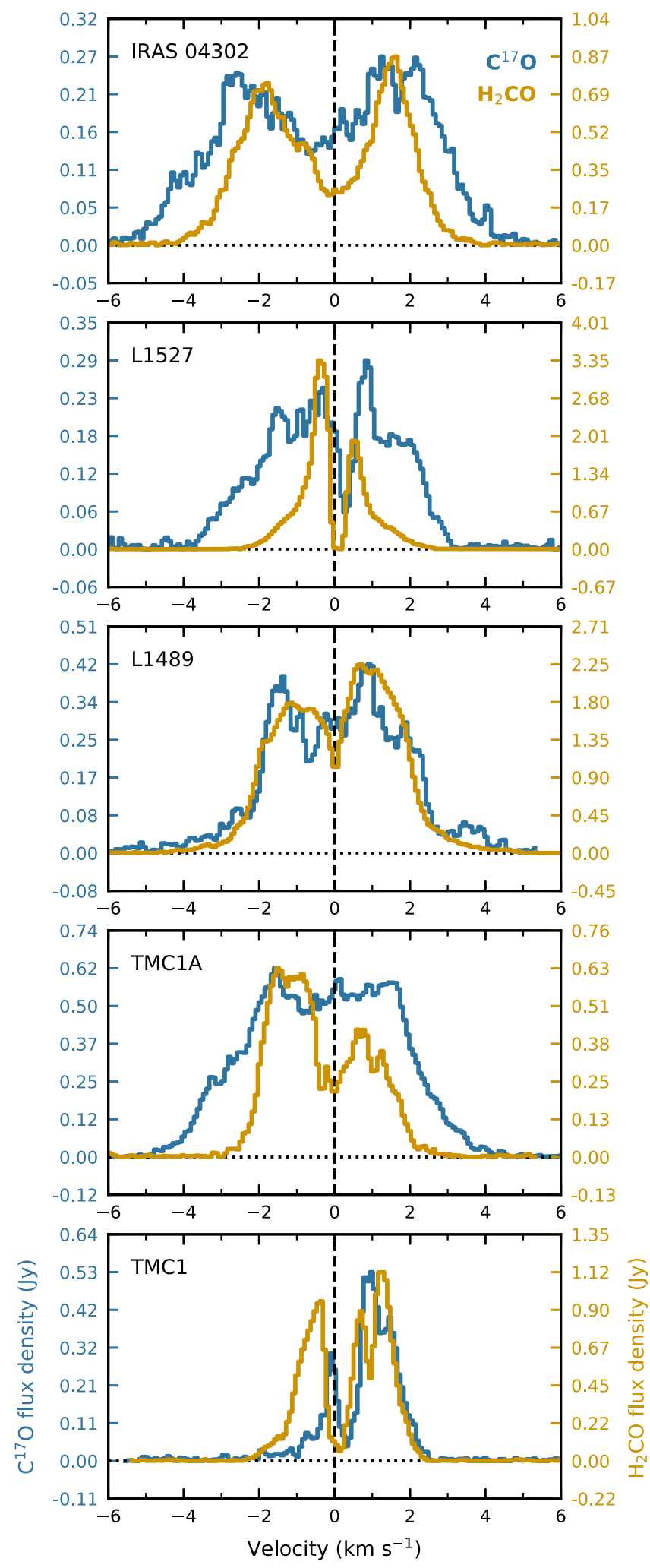

Figure A2. Spectra for $\mathrm{C}^{17} \mathrm{O}$ (blue) and $\mathrm{H}_{2} \mathrm{CO}$ (orange) extracted in a $6^{\prime \prime}$ circular aperture centered at the continuum peak. Only pixels with $>3 \sigma$ emission are included. The vertical scale is different for each molecular line in each panel. The vertical dashed lines mark the systemic velocities, which have been shifted to $0 \mathrm{~km} \mathrm{~s}^{-1}$.

\section{Appendix B \\ Envelope Contribution}

A first assessment of the envelope contribution to the line emission can be made by comparing generic models of either a Keplerian disk only or a disk embedded in an envelope to the observed visibility amplitudes. To do so, we calculated the visibility amplitude profiles for a Keplerian disk in $0.5 \mathrm{~km} \mathrm{~s}^{-1}$ channels using the modeling tools outlined in Sheehan et al. (2019). Values for the stellar mass, disk radius, inclination, and position angle were adopted from the 
IRAS $04302-\mathrm{C}^{17} \mathrm{O}$

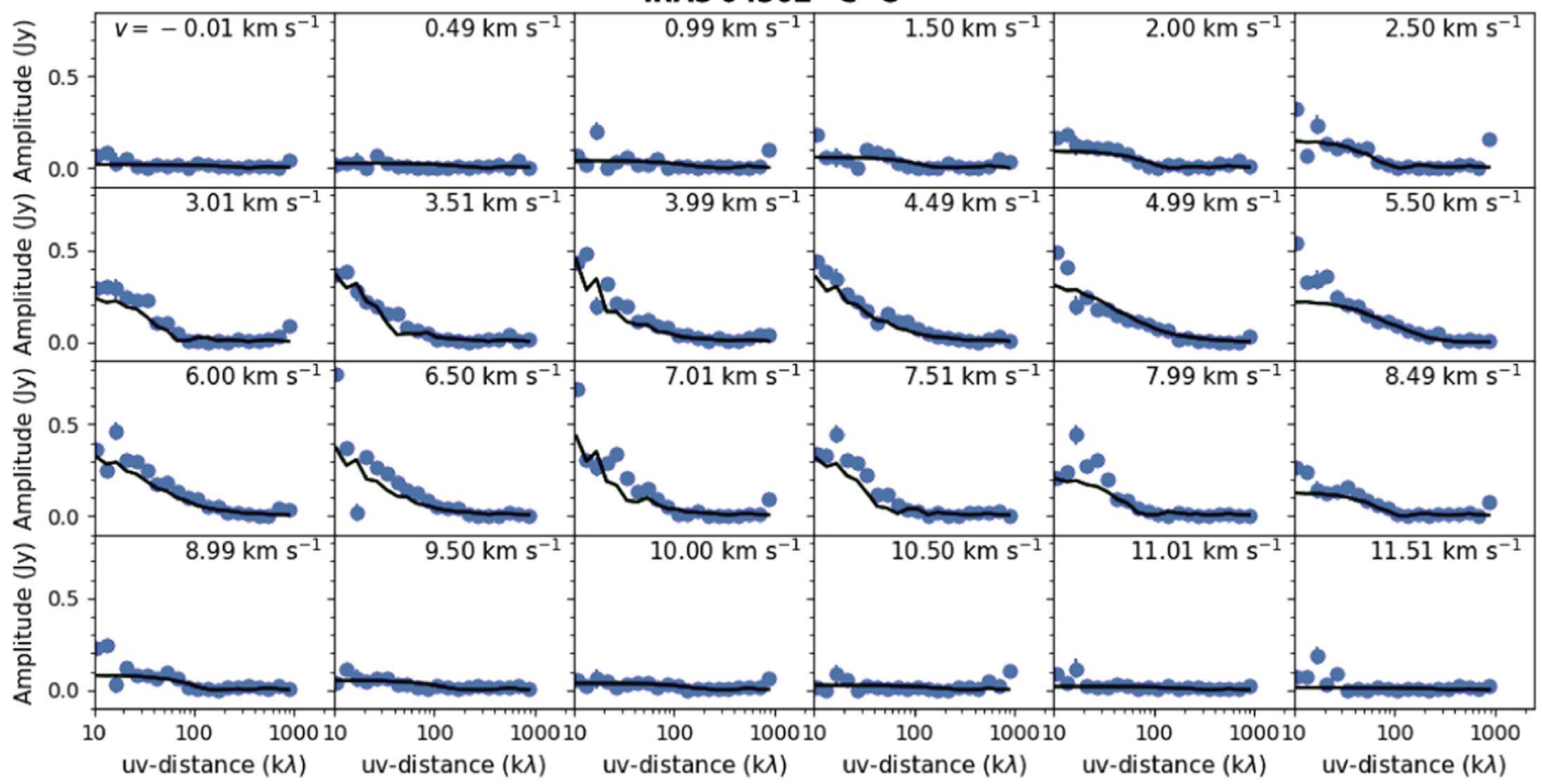

TMC1A - C ${ }^{17} \mathrm{O}$

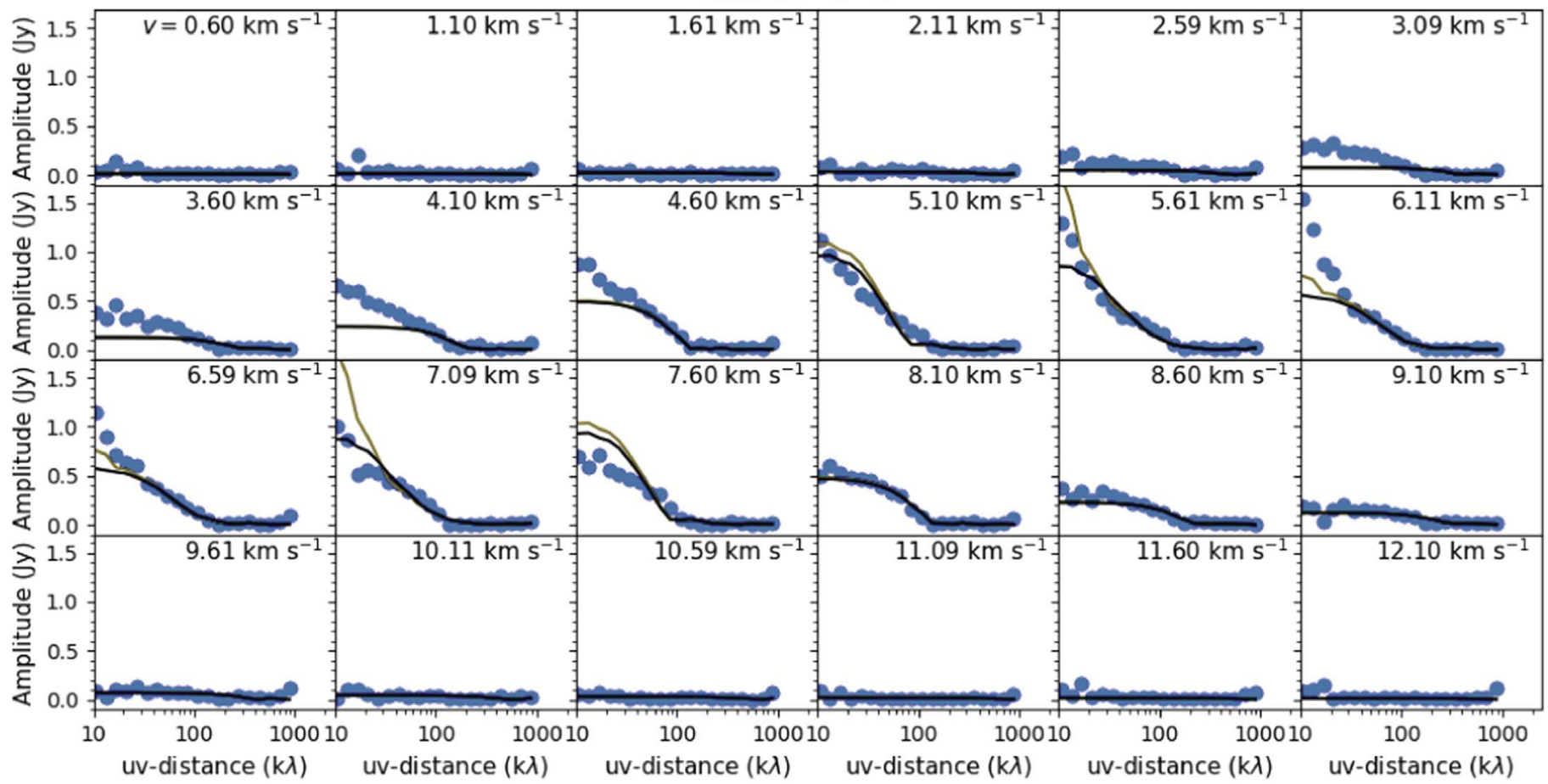

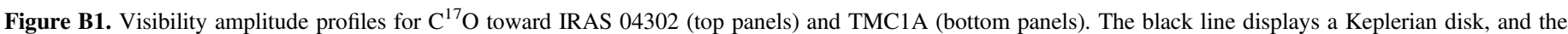

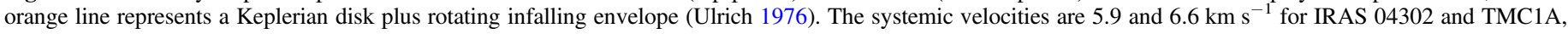
respectively.

literature, and the $\mathrm{C}^{17} \mathrm{O}$ and $\mathrm{H}_{2} \mathrm{CO}$ abundances were taken as constant throughout the disk. The disk mass was adjusted to approximately match the visibility amplitude profiles in each channel. If there was a component at small uv-distances that could not be reproduced with the disk, we added a rotating infalling envelope with a 3000 au radius using the prescription by Ulrich (1976). The results for $\mathrm{C}^{17} \mathrm{O}$ toward
IRAS 04302 and TMC1A are shown as an example in Figure B1. We stress that we do not expect a perfect fit with this simple approach, but it shows that the $\mathrm{C}^{17} \mathrm{O}$ emission toward IRAS 04302 can be reproduced without an envelope, while some envelope contribution is required at low velocities $\left(\sim|1| \mathrm{km} \mathrm{s}^{-1}\right.$ from the systemic velocity) toward TMC1A. 
warm

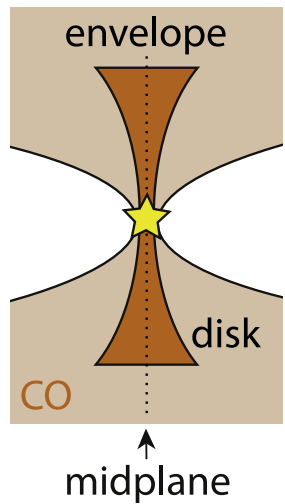

\section{intermediate}

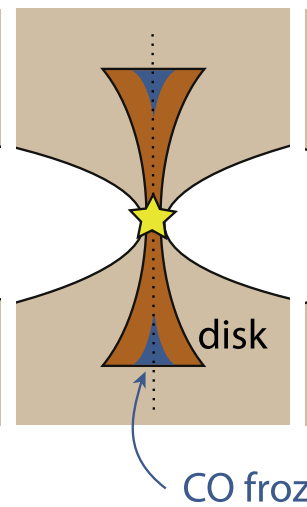

\section{cold}

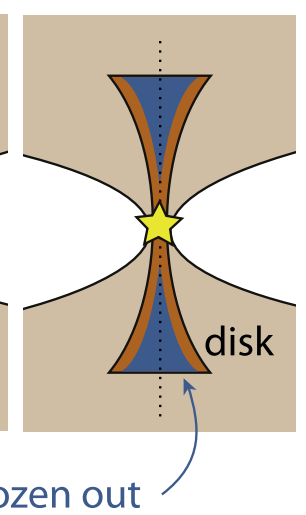

Figure C1. Three different models for the CO distribution in embedded disks. Left panel: warm disk with no CO freeze-out. Middle panel: slightly colder disk where $\mathrm{CO}$ is frozen out in the outer disk midplane. Right panel: cold disk where gaseous $\mathrm{CO}$ is only present in the inner disk and disk surface layers. Gaseous CO is present in the inner envelope in all models. Figure reproduced from van 't Hoff et al. (2018a).

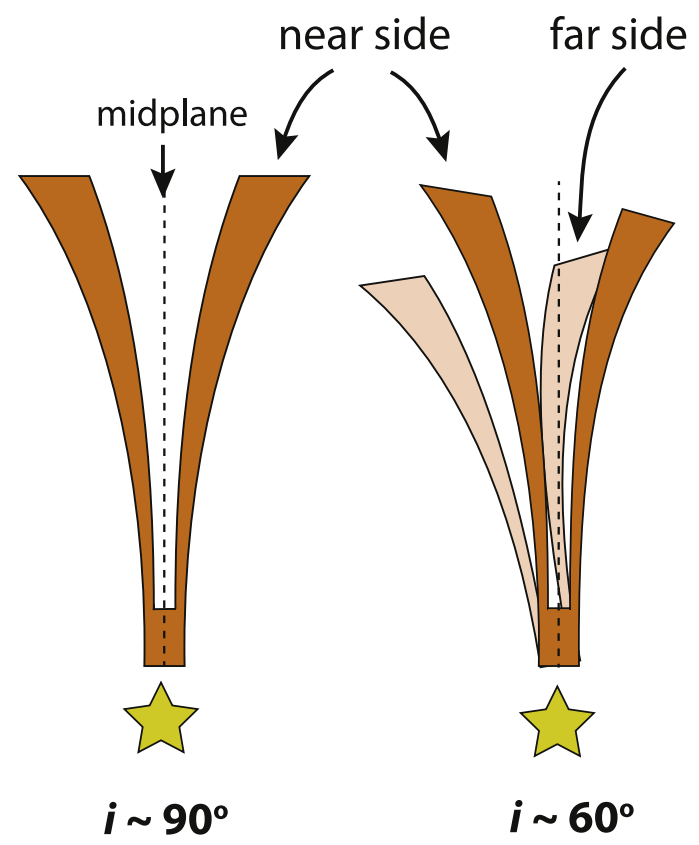

Figure C2. Schematic representation of a disk with emission originating only in the surface layers viewed edge-on $\left(\sim 90^{\circ}\right.$; left panel) and at an inclination of $\sim 60^{\circ}$ (right panel). In the edge-on orientation, only the near side of the disk is visible, and at sufficient angular resolution, a V-shaped emission pattern is observed. In contrast, when the disk is $\sim 60^{\circ}$ inclined, the far side of the disk becomes visible, and emission from the far side appears to be coming from the midplane. This is especially problematic at low angular resolution, when the continuum disk is too small to map out the midplane or the line is too weak to be detected in individual channels at high enough spectral resolution.

\section{Appendix C Schematics of the Disk Models}

Figure $\mathrm{C} 1$ shows a schematic overview of the warm, intermediate, and cold disk models as presented by van 't Hoff et al. (2018a). In the warm model, CO is present in the gas phase in the entire disk, whereas in the cold model, $\mathrm{CO}$ is frozen out in most of the disk, with gas-phase $\mathrm{CO}$ only present in the inner disk and disk surface layers. In the intermediate model, $\mathrm{CO}$ freeze-out occurs in the outer midplane. A constant gas-phase $\mathrm{CO}$ abundance of $10^{-4}$ with respect to $\mathrm{H}_{2}$ is adopted in the regions where $T>20 \mathrm{~K}$. If the envelope is included in the radiative transfer, gas-phase $\mathrm{CO}$ is present in the $T>20 \mathrm{~K}$ region at an abundance of $10^{-4}$ as well. For the physical structure (dust density and temperature), we adopt the model for L1527 from Tobin et al. (2013), who modeled the disk continuum emission by fitting both the visibilities and images of $870 \mu \mathrm{m}$ and $3.4 \mathrm{~mm}$ observations, the multiwavelength spectral energy distribution, and $L^{\prime}$ scattered-light images with $3 \mathrm{D}$ radiative transfer modeling.

Figure C2 illustrates why observing freeze-out directly can be challenging in disks that are not viewed edge-on.

\section{ORCID iDs}

Merel L. R. van 't Hoff (1) https://orcid.org/0000-00022555-9869

Daniel Harsono (ㄱ https://orcid.org/0000-0001-6307-4195

John J. Tobin (1) https://orcid.org/0000-0002-6195-0152

Arthur D. Bosman (1) https://orcid.org/0000-0003-4001-3589

Ewine F. van Dishoeck (1) https://orcid.org/0000-00017591-1907

Jes K. Jørgensen (1) https://orcid.org/0000-0001-9133-8047

Anna Miotello (ㄴ) https://orcid.org/0000-0002-7997-2528

Catherine Walsh ํㅏ https://orcid.org/0000-0001-6078-786X

\section{References}

Adams, F. C., \& Shu, F. H. 1986, ApJ, 308, 836

Aikawa, Y., Umebayashi, T., Nakano, T., \& Miyama, S. M. 1999, ApJ, 519, 705

Aikawa, Y., van Zadelhoff, G. J., van Dishoeck, E. F., \& Herbst, E. 2002, A\&A, 386, 622

Ali-Dib, M. 2017, MNRAS, 467, 2845

ALMA Partnership, Brogan, C. L., Pérez, L. M., et al. 2015, ApJL, 808, L3

Andersen, B. C., Stephens, I. W., Dunham, M. M., et al. 2019, ApJ, 873, 54

Andrews, S. M., Huang, J., Pérez, L. M., et al. 2018, ApJL, 869, L41

Andrews, S. M., Wilner, D. J., Hughes, A. M., Qi, C., \& Dullemond, C. P. 2010, ApJ, 723, 1241

Ansdell, M., Williams, J. P., Manara, C. F., et al. 2017, AJ, 153, 240

Ansdell, M., Williams, J. P., van der Marel, N., et al. 2016, ApJ, 828, 46

Artur de la Villarmois, E., Jørgensen, J. K., Kristensen, L. E., et al. 2019, A\&A, 626, A71

Aso, Y., Ohashi, N., Aikawa, Y., et al. 2017, ApJ, 849, 56

Aso, Y., Ohashi, N., Saigo, K., et al. 2015, ApJ, 812, 27

Barenfeld, S. A., Carpenter, J. M., Ricci, L., \& Isella, A. 2016, ApJ, 827, 142 Bergin, E. A., Cleeves, L. I., Crockett, N., \& Blake, G. A. 2014, FaDi, 168, 61 Bergner, J. B., Guzmán, V. G., Öberg, K. I., Loomis, R. A., \& Pegues, J. 2018, ApJ, 857, 69

Bergner, J. B., Oberg, K. I., Bergin, E. A., et al. 2020, ApJ, 898, 97

Bertin, M., Romanzin, C., Doronin, M., et al. 2016, ApJL, 817, L12

Boley, A. C. 2009, ApJL, 695, L53

Boogert, A. C. A., Gerakines, P. A., \& Whittet, D. C. B. 2015, ARA\&A, 53,541

Bosman, A. D., Walsh, C., \& van Dishoeck, E. F. 2018, A\&A, 618, A182

Boss, A. P. 1997, Sci, 276, 1836

Brinch, C., Crapsi, A., Jørgensen, J. K., Hogerheijde, M. R., \& Hill, T. 2007, A\&A, 475, 915

Brinch, C., \& Hogerheijde, M. R. 2010, A\&A, 523, A25

Carney, M. T., Hogerheijde, M. R., Guzmán, V. V., et al. 2019, A\&A, 623, A124

Carney, M. T., Hogerheijde, M. R., Loomis, R. A., et al. 2017, A\&A, 605, A21 Chiang, E. I., \& Goldreich, P. 1997, ApJ, 490, 368

Chuang, K.-J., Fedoseev, G., Ioppolo, S., van Dishoeck, E. F., \& Linnartz, H. 2016, MNRAS, 455, 1702

Cieza, L. A., Ruíz-Rodríguez, D., Hales, A., et al. 2019, MNRAS, 482, 698

Cleeves, L. I. 2016, ApJL, 816, L21

Cleeves, L. I., Bergin, E. A., Qi, C., Adams, F. C., \& Öberg, K. I. 2015, ApJ, 799, 204

Cox, E. G., Harris, R. J., Looney, L. W., et al. 2017, ApJ, 851, 83

Cridland, A. J., Eistrup, C., \& van Dishoeck, E. F. 2019, A\&A, 627, A127 
Cruz-Diaz, G. A., Martín-Doménech, R., Muñoz Caro, G. M., \& Chen, Y. J. 2016, A\&A, 592, A68

Cuppen, H. M., van Dishoeck, E. F., Herbst, E., \& Tielens, A. G. G. M. 2009, A\&A, 508, 275

Curran, R. L., Argiroffi, C., Sacco, G. G., et al. 2011, A\&A, 526, A104

D’Alessio, P., Calvet, N., \& Hartmann, L. 1997, ApJ, 474, 397

Drążkowska, J., \& Alibert, Y. 2017, A\&A, 608, A92

Drozdovskaya, M. N., Walsh, C., Visser, R., Harsono, D., \& van Dishoeck, E. F. 2014, MNRAS, 445, 913

Dutrey, A., Guilloteau, S., \& Guelin, M. 1997, A\&A, 317, L55

Dutrey, A., Guilloteau, S., Piétu, V., et al. 2017, A\&A, 607, 130

Eistrup, C., Walsh, C., \& van Dishoeck, E. F. 2016, A\&A, 595, A83

Facchini, S., Birnstiel, T., Bruderer, S., \& van Dishoeck, E. F. 2017, A\&A, 605, A16

Favre, C., Cleeves, L. I., Bergin, E. A., Qi, C., \& Blake, G. A. 2013, ApJL, 776, L38

Favre, C., Fedele, D., Semenov, D., et al. 2018, ApJL, 862, L2

Fedoseev, G., Cuppen, H. M., Ioppolo, S., Lamberts, T., \& Linnartz, H. 2015, MNRAS, 448, 1288

Frimann, S., Jørgensen, J. K., Dunham, M. M., et al. 2017, A\&A, 602, A120

Fuchs, G. W., Cuppen, H. M., Ioppolo, S., et al. 2009, A\&A, 505, 629

Furuya, K., \& Aikawa, Y. 2014, ApJ, 790, 97

Garrod, R. T., \& Herbst, E. 2006, A\&A, 457, 927

Green, J. D., Evans, N. J. I., Jørgensen, J. K., et al. 2013, ApJ, 770, 123

Harsono, D., Bjerkeli, P., van der Wiel, M. H. D., et al. 2018, NatAs, 2, 646

Harsono, D., Bruderer, S., \& van Dishoeck, E. F. 2015, A\&A, 582, A41

Harsono, D., Jørgensen, J. K., van Dishoeck, E. F., et al. 2014, A\&A, 562, A77

Hartmann, L., Herczeg, G., \& Calvet, N. 2016, ARA\&A, 54, 135

Henning, T., \& Semenov, D. 2008, in IAU Symp. 251, Organic Matter in Space, ed. S. Kwok \& S. Sanford (Cambridge: Cambridge Univ. Press), 89

Herczeg, G. J., \& Hillenbrand, L. A. 2008, ApJ, 681, 594

Hildebrand, R. H. 1983, QJRAS, 24, 267

Hsieh, T.-H., Murillo, N. M., Belloche, A., et al. 2019, ApJ, 884, 149

Huang, J., Öberg, K. I., Qi, C., et al. 2017, ApJ, 835, 231

Ingleby, L., Calvet, N., Herczeg, G., et al. 2013, ApJ, 767, 112

Jørgensen, J. K., Bourke, T. L., Myers, P. C., et al. 2007, ApJ, 659, 479

Jørgensen, J. K., van der Wiel, M. H. D., Coutens, A., et al. 2016, A\&A, 595, A117

Jørgensen, J. K., van Dishoeck, E. F., Visser, R., et al. 2009, A\&A, 507, 861

Jørgensen, J. K., Visser, R., Williams, J. P., \& Bergin, E. A. 2015, A\&A, 579, A23

Kama, M., Bruderer, S., van Dishoeck, E. F., et al. 2016, A\&A, 592, A83

Karska, A., Kaufman, M. J., Kristensen, L. E., et al. 2018, ApJS, 235, 30

Kenyon, S. J., Calvet, N., \& Hartmann, L. 1993, ApJ, 414, 676

Kratter, K., \& Lodato, G. 2016, ARA\&A, 54, 271

Krijt, S., Schwarz, K. R., Bergin, E. A., \& Ciesla, F. J. 2018, ApJ, 864, 78

Kristensen, L. E., van Dishoeck, E. F., Bergin, E. A., et al. 2012, A\&A, 542 , A8

Kwon, W., Looney, L. W., Mundy, L. G., Chiang, H.-F., \& Kemball, A. J. 2009, ApJ, 696, 841

Lee, J.-E., Lee, S., Baek, G., et al. 2019, NatAs, 3, 314

Long, F., Herczeg, G. J., Pascucci, I., et al. 2017, ApJ, 844, 99

Loomis, R. A., Cleeves, L. I., Öberg, K. I., et al. 2018, ApJ, 859, 131

Loomis, R. A., Cleeves, L. I., Öberg, K. I., Guzman, V. V., \& Andrews, S. M. 2015, ApJL, 809, L25

Madhusudhan, N., Amin, M. A., \& Kennedy, G. M. 2014, ApJL, 794, L12

Manara, C. F., Morbidelli, A., \& Guillot, T. 2018, A\&A, 618, L3

Manara, C. F., Rosotti, G., Testi, L., et al. 2016, A\&A, 591, L3

Mangum, J. G., \& Wootten, A. 1993, ApJS, 89, 123

Mathews, G. S., Klaassen, P. D., Juhász, A., et al. 2013, A\&A, 557, A132

McClure, M. K., Bergin, E. A., Cleeves, L. I., et al. 2016, ApJ, 831, 167

McElroy, D., Walsh, C., Markwick, A. J., et al. 2013, A\&A, 550, A36

McMullin, J. P., Waters, B., Schiebel, D., Young, W., \& Golap, K. 2007, in ASP Conf. Ser. 376, Astronomical Data Analysis Software and Systems XVI, ed. R. A. Shaw, F. Hill, \& D. J. Bell (San Francisco, CA: ASP), 127

Miotello, A., Testi, L., Lodato, G., et al. 2014, A\&A, 567, A32

Miotello, A., van Dishoeck, E. F., Williams, J. P., et al. 2017, A\&A, 599, A113

Motte, F., \& André, P. 2001, A\&A, 365, 440

Mottram, J. C., van Dishoeck, E. F., Kristensen, L. E., et al. 2017, A\&A, 600, A99

Müller, H. S. P., Schlöder, F., Stutzki, J., \& Winnewisser, G. 2005, JMoSt, 742,215

Murillo, N. M., Bruderer, S., van Dishoeck, E. F., et al. 2015, A\&A, 579, A114

Murillo, N. M., Lai, S.-P., Bruderer, S., Harsono, D., \& van Dishoeck, E. F. 2013, A\&A, 560, A103
Noble, J. A., Theule, P., Mispelaer, F., et al. 2012, A\&A, 543, A5

Öberg, K. I., Furuya, K., Loomis, R., et al. 2015, ApJ, 810, 112

Öberg, K. I., Guzmán, V. V., Merchantz, C. J., et al. 2017, ApJ, 839, 43

Öberg, K. I., Murray-Clay, R., \& Bergin, E. A. 2011, ApJL, 743, L16

Öberg, K. I., Qi, C., Fogel, J. K. J., et al. 2010, ApJ, 720, 480

Ohashi, N., Saigo, K., Aso, Y., et al. 2014, ApJ, 796, 131

Oya, Y., Sakai, N., Lefloch, B., et al. 2015, ApJ, 812, 59

Oya, Y., Sakai, N., López-Sepulcre, A., et al. 2016, ApJ, 824, 88

Palla, F., \& Stahler, S. W. 1993, ApJ, 418, 414

Pascucci, I., Testi, L., Herczeg, G. J., et al. 2016, ApJ, 831, 125

Pegues, J., Öberg, K. I., Bergner, J. B., et al. 2020, ApJ, 890, 142

Persson, M. V., Jørgensen, J. K., \& van Dishoeck, E. F. 2013, A\&A, 549, L3

Persson, M. V., Jørgensen, J. K., van Dishoeck, E. F., \& Harsono, D. 2014, A\&A, 563, A74

Pickett, H. M., Poynter, R. L., Cohen, E. A., et al. 1998, JQSRT, 60, 883

Pinte, C., Ménard, F., Duchêne, G., et al. 2018, A\&A, 609, A47

Podio, L., Bacciotti, F., Fedele, D., et al. 2019, A\&A, 623, L6

Qi, C., Öberg, K. I., Andrews, S. M., et al. 2015, ApJ, 813, 128

Qi, C., Öberg, K. I., Espaillat, C. C., et al. 2019, ApJ, 882, 160

Qi, C., Öberg, K. I., \& Wilner, D. J. 2013a, ApJ, 765, 34

Qi, C., Öberg, K. I., Wilner, D. J., et al. 2013b, Sci, 341, 630

Ruíz-Rodríguez, D., Cieza, L. A., Williams, J. P., et al. 2018, MNRAS, 478,3674

Sai, J., Ohashi, N., Saigo, K., et al. 2020, ApJ, 893, 51

Sakai, N., Oya, Y., Sakai, T., et al. 2014a, ApJL, 791, L38

Sakai, N., Sakai, T., Hirota, T., et al. 2014b, Natur, 507, 78

Schöier, F. L., Jørgensen, J. K., van Dishoeck, E. F., \& Blake, G. A. 2002, A\&A, 390, 1001

Schöier, F. L., van der Tak, F. F. S., van Dishoeck, E. F., \& Black, J. H. 2005, A\&A, 432, 369

Schoonenberg, D., \& Ormel, C. W. 2017, A\&A, 602, A21

Schwarz, K. R., Bergin, E. A., Cleeves, L. I., et al. 2016, ApJ, 823, 91

Schwarz, K. R., Bergin, E. A., Cleeves, L. I., et al. 2018, ApJ, 856, 85

Schwarz, K. R., Bergin, E. A., Cleeves, L. I., et al. 2019, ApJ, 877, 131

Sheehan, P. D., \& Eisner, J. A. 2017, ApJ, 851, 45

Sheehan, P. D., Wu, Y.-L., Eisner, J. A., \& Tobin, J. J. 2019, ApJ, 874, 136

Stahler, S. W., Shu, F. H., \& Taam, R. E. 1980, ApJ, 241, 637

Stevenson, D. J., \& Lunine, J. I. 1988, Icar, 75, 146

Taquet, V., López-Sepulcre, A., Ceccarelli, C., et al. 2013, ApJL, 768, L29

Taquet, V., López-Sepulcre, A., Ceccarelli, C., et al. 2015, ApJ, 804, 81

Thi, W.-F., van Zadelhoff, G.-J., \& van Dishoeck, E. F. 2004, A\&A, 425, 955

Tielens, A. G. G. M., \& Hagen, W. 1982, A\&A, 114, 245

Tobin, J. J., Hartmann, L., Calvet, N., \& D’Alessio, P. 2008, ApJ, 679, 1364

Tobin, J. J., Hartmann, L., Chiang, H.-F., et al. 2012, Natur, 492, 83

Tobin, J. J., Hartmann, L., Chiang, H.-F., et al. 2013, ApJ, 771, 48

Tobin, J. J., Kratter, K. M., Persson, M. V., et al. 2016a, Natur, 538, 483

Tobin, J. J., Looney, L. W., Li, Z.-Y., et al. 2016b, ApJ, 818, 73

Tobin, J. J., Looney, L. W., Wilner, D. J., et al. 2015, ApJ, 805, 125

Tobin, J. J., Sheehan, P. D., Megeath, S. T., et al. 2020, ApJ, 890, 130

Tychoniec, Ł., Manara, C. F., Rosotti, G. P., et al. 2020, A\&A, 640, A19

Tychoniec, Ł., Tobin, J. J., Karska, A., et al. 2018, ApJS, 238, 19

Ulrich, R. K. 1976, ApJ, 210, 377

van Gelder, M. L., Tabone, B., Tychoniec, L., et al. 2020, A\&A, 639, 87

van 't Hoff, M. 2019, PhD thesis, Leiden Univ.

van 't Hoff, M. L. R., Tobin, J. J., Harsono, D., \& van Dishoeck, E. F. 2018a, A\&A, 615, A83

van 't Hoff, M. L. R., Tobin, J. J., Trapman, L., et al. 2018b, ApJL, 864, L23

van 't Hoff, M. L. R., van Dishoeck, E. F., Jørgensen, J. K., \& Calcutt, H. 2020, A\&A, 633, A7

van 't Hoff, M. L. R., Walsh, C., Kama, M., Facchini, S., \& van Dishoeck, E. F. 2017, A\&A, 599, A101

van Zadelhoff, G.-J., van Dishoeck, E. F., Thi, W.-F., \& Blake, G. A. 2001, A\&A, 377, 566

Visser, R., Doty, S. D., \& van Dishoeck, E. F. 2011, A\&A, 534, A132

Visser, R., Kristensen, L. E., Bruderer, S., et al. 2012, A\&A, 537, A55

Visser, R., van Dishoeck, E. F., Doty, S. D., \& Dullemond, C. P. 2009, A\&A, 495,881

Vorobyov, E. I. 2009, ApJ, 704, 715

Walsh, C., Loomis, R. A., Öberg, K. I., et al. 2016, ApJL, 823, L10

Walsh, C., Millar, T. J., Nomura, H., et al. 2014, A\&A, 563, A33

Walsh, C., Nomura, H., \& van Dishoeck, E. 2015, A\&A, 582, A88

Watanabe, N., \& Kouchi, A. 2002, ApJL, 571, L173

Whitney, B. A., Wood, K., Bjorkman, J. E., \& Wolff, M. J. 2003, ApJ, 591, 1049

Willacy, K., \& Woods, P. M. 2009, ApJ, 703, 479 
Williams, J. P., Cieza, L., Hales, A., et al. 2019, ApJL, 875, L9

Wilson, T. L., \& Rood, R. 1994, ARA\&A, 32, 191

Wolf, S., Padgett, D. L., \& Stapelfeldt, K. R. 2003, ApJ, 588, 373

Xu, R., Bai, X.-N., \& Öberg, K. 2017, ApJ, 835, 162

Yen, H.-W., Koch, P. M., Takakuwa, S., et al. 2017, ApJ, 834, 178

Yen, H.-W., Takakuwa, S., Ohashi, N., et al. 2014, ApJ, 793, 1
Yen, H.-W., Takakuwa, S., Ohashi, N., \& Ho, P. T. P. 2013, ApJ, 772, 22

Yıldız, U. A., Kristensen, L. E., van Dishoeck, E. F., et al. 2015, A\&A, 576, A109

Zhang, K., Bergin, E. A., Blake, G. A., Cleeves, L. I., \& Schwarz, K. R. 2017, NatAs, 1, 0130

Zhang, K., Schwarz, K. R., \& Bergin, E. A. 2020, ApJL, 891, L17 